\title{
On fluid flows in precessing narrow annular channels: asymptotic analysis and numerical simulation
}

\author{
KEKE ZHANG ${ }^{1} \dagger$, DALI KONG ${ }^{1}$ AND XINHAO LIAO \\ ${ }^{1}$ Department of Mathematical Sciences, University of Exeter, EX4 4QF, UK \\ ${ }^{2}$ Shanghai Astronomical Observatory, Chinese Academy of Sciences, Shanghai 200030, China
}

(Received 17 July 2009; revised 20 February 2010; accepted 24 February 2010; first published online 20 May 2010)

We consider a viscous, incompressible fluid confined in a narrow annular channel rotating rapidly about its axis of symmetry with angular velocity $\boldsymbol{\Omega}$ that itself precesses slowly about an axis fixed in an inertial frame. The precessional problem is characterized by three parameters: the Ekman number $E$, the Poincare number $\epsilon$ and the aspect ratio of the channel $\Gamma$. Dependent upon the size of $\Gamma$, precessionally driven flows can be either resonant or non-resonant with the Poincare forcing. By assuming that it is the viscous effect, rather than the nonlinear effect, that plays an essential role at exact resonance, two asymptotic expressions for $\epsilon \ll 1$ and $E \ll 1$ describing the single and double inertial-mode resonance are derived under the nonslip boundary condition. An asymptotic expression describing non-resonant precessing flows is also derived. Further studies based on numerical integrations, including twodimensional linear analysis and direct three-dimensional nonlinear simulation, show a satisfactory quantitative agreement between the three asymptotic expressions and the fuller numerics for small and moderate Reynolds numbers at an asymptotically small $E$. The transition from two-dimensional precessing flow to three-dimensional small-scale turbulence for large Reynolds numbers is also investigated.

\section{Introduction}

It has been conjectured that precessionally driven flows in the Earth's fluid core may be sufficiently strong and complex to be responsible for generating and maintaining the geomagnetic field (Bullard 1949). This conjecture has been supported by laboratory and numerical experiments demonstrating that wave-like instabilities and transition to turbulent flows can occur in precessing spherical/spheroidal systems (see, for example, Malkus 1968; Kerswell 1993; Vanyo et al. 1995; Hollerbach \& Kerswell 1995; Tilgner \& Busse 2001; Noir, Jault, \& Cardin 2001; Noir et al. 2003) and in precessing cylinders (see, for example, Gans 1970; Malkus 1989; Manasseh 1992; Kobine 1995; Meunier et al. 2008), by the theoretical estimate of abundant precessional energy (see, for example, Kerswell 1996) and by convincing numerical experiments showing that precession-driven flows can indeed generate and sustain magnetic fields (see, for example, Tilgner 2005, 2007; Wu \& Roberts 2008, 2009). Although the time scale of precessionally driven flows is usually much shorter compared with that of planetary 
convection, the persistent nature of precession makes it significant in many problems of geophysical and astrophysical fluid dynamics.

Motivated by the desire to understand the fundamental dynamics and physics, Mason \& Kerswell (2002) carried out an important study of precessionally driven flow in planar geometry, the simplest of all existing models of the precessional problem. They considered a viscous, incompressible fluid confined between two infinitely extended parallel plane boundaries that rotate rapidly about their normal with angular velocity $\boldsymbol{\Omega}$ that itself precesses slowly about a horizontal axis fixed in space with angular velocity $\boldsymbol{\Omega}_{p}$. Note that there is no essential loss of generality in assuming that $\boldsymbol{\Omega}$ is perpendicular to $\boldsymbol{\Omega}_{p}$ because any component of $\boldsymbol{\Omega}_{p}$ parallel to $\boldsymbol{\Omega}$ may be absorbed into a redefined $\boldsymbol{\Omega}(\mathrm{Wu} \&$ Roberts 2009$)$. In unbounded planar geometry, the precessional motion of the two parallel boundaries drives flows against viscous dissipation. When the precessional rate increases, their numerical simulation shows that the precessionally driven flow changes from two-dimensional laminar to three-dimensional chaotic. To avoid the thin viscous boundary layers on the top and bottom of the plane layer, which are numerically expensive to resolve, the stress-free boundary condition is employed in their numerical study. In a further development of the same planar geometry model, Wu \& Roberts (2008) include the effects of a self-generated magnetic field, demonstrating that, if the fluid is sufficiently electrically conducting, the precessing flow at a sufficiently large precessional rate can support dynamo action.

Motivated by possible geophysical and astrophysical applications, annular geometry has been widely employed to study/mimic fluid motion taking place in the equatorial region or low latitudes of rotating spherical shells (see, for example, Gilman 1973; Busse 1994; Jones, Rotvig \& Abdulrahman 2003; Eccles et al. 2009). There are several reasons why annular geometry has been used in modelling rotating flows. First, in comparison to either spherical-shell geometry or unbounded planar geometry, the rotating annular configuration is readily experimentally realizable (see, for example, Davies-Jones \& Gilman 1971; Busse 1994; Eccles et al. 2009). Second, as a consequence of rapid rotation, the key dynamics in the equatorial region would be different from that in the polar regions in rotating spherical shells. Third, the mathematical degeneracy characterizing unbounded planar geometry is removed by the presence of two lateral sidewalls (see, for example, Davies-Jones \& Gilman 1971). At the same time, the mathematical simplicity and clarity of planar geometry - using local Cartesian coordinates - remain unchanged in annular geometry when the gap width of an annulus is sufficiently small in comparison with its radius. This narrow-gap approximation has been usually employed in the theoretical studies of fluid dynamics in a rotating annulus (see, for example, Davies-Jones \& Gilman 1971; Jones et al. 2003; Busse 2005). A major advantage of neglecting the curvature effect in a narrow annulus is that a relatively simple analytical description of fluid motion is permitted, offering a helpful insight into the essential dynamics of rotating flows (see, for example, Busse 2005; Liao, Zhang \& Chang 2006). This approximation is supported by both the numerical and experimental studies on convection in a rotating annulus, demonstrating that the primary features of the flows in a narrow-gap annulus with the curvature effect are captured by a narrow-gap annulus that uses the narrow-gap approximation to neglect the curvature effect (see, for example, Busse 1994; Li et al. 2008). Although this study is primarily motivated by possible geophysical and astrophysical applications, the problem of precessionally driven flows in annular/cylindrical geometry may have industrial applications because propellant tanks in a flying spacecraft can be subject to the precessional forcing (see, for example, Vanyo 1993). 
The primary objective of the present study is to understand, through both asymptotic and numerical analyses, the fundamental dynamics of the precessionally driven flow of a viscous, incompressible fluid confined in a rotating narrow annulus using the narrow-gap approximation, the same geometry proposed by Davies-Jones \& Gilman (1971) and Gilman (1973) (see also Busse 2005). The narrow annular channel rotates rapidly about its axis of symmetry with angular velocity $\Omega$ that itself precesses slowly about an axis that is fixed in space and perpendicular to $\Omega$. A non-slip velocity boundary condition, appropriate for experimental studies of the problem, will be used. A major advantage of the narrow annular configuration is that it is approximately realizable in laboratory experiments (Davies-Jones \& Gilman 1971; Gilman 1973). A major disadvantage of the realistic non-slip condition is that the thin Ekman boundary layers are numerically expensive to resolve. This is, however, rewarded by the benefits that the non-slip precessing solutions are directly comparable with experimental results and, perhaps more significantly, that the Ekman boundary layers associated with the non-slip condition play a central role at resonance (Roberts \& Stewartson 1965).

In addition to the Ekman number $E$ and the Poincare number $\epsilon$ in the planar precessional problem (Mason \& Kerswell 2002; Wu \& Roberts 2008), the existence of four rigid walls in channel geometry not only introduces a new parameter $\Gamma$, the aspect ratio of the depth to the width of a channel, but also introduces new dynamics into the precessional problem. Depending upon the size of the aspect ratio $\Gamma$, the precessing flow in a channel can be divided into three different categories: (a) a single principal inertial mode is at exact resonance with the Poincare forcing; (b) two principal inertial modes are at exact resonance with the Poincare forcing; and (c) multiple inertial modes are excited at non-resonance. When the precessional forcing is small, we shall derive three asymptotic expressions that describe precessionally driven flows of the three different types in a channel with the non-slip boundary condition. We shall demonstrate that the viscous boundary layers, in connection with the non-slip boundary condition, are physically and mathematically important in determining the primary properties, such as the amplitude, of a precessing flow at exact resonance. We shall also perform two-dimensional linear numerical analysis and fully three-dimensional nonlinear simulation, showing a satisfactory quantitative agreement between the asymptotic expressions and the numerical analyses for small and moderate Reynolds numbers. Moreover, we shall study the transition from two-dimensional precessing flow to three-dimensional small-scale turbulence for large Reynolds numbers, revealing a breakdown of the laminar flow to small-scale turbulence at a moderate precessional rate at exact resonance.

In what follows we shall begin by presenting the mathematical equations of the problem in $\S 2$. The asymptotic analysis for the three types of precessionally driven flows is discussed in $\S 3$ while the two-dimensional linear numerical analysis and three-dimensional nonlinear simulation are presented in $\S 4$. A summary and some remarks are given in $\S 5$.

\section{Mathematical formulation}

Consider a viscous, incompressible fluid occupying an annular channel with inner radius $r_{i} d$, outer radius $r_{o} d$ and depth $d$. A parameter $\Gamma$ is introduced to denote the aspect ratio of the channel $\Gamma=\left(r_{o} d-r_{i} d\right) / d$. When the gap of the annulus is sufficiently small, i.e. $\Gamma / r_{o} \ll 1$, which will be referred to as a narrow annular channel (Busse 2005), the effect of the curvature can be neglected by using the small-gap 
approximation with the periodic condition along the channel (Gilman 1973; Busse 2005). The configuration of the narrow annular channel will be adopted in the present study of precessionally driven flows.

The narrow annular channel rotates rapidly with angular velocity $\hat{z} \Omega$ about its axis of symmetry and precesses slowly about an axis that is perpendicular to $\hat{z}$ and fixed in space, which is similar to that in the precessional problem of planar geometry (Mason \& Kerswell 2002; Wu \& Roberts 2008). In this paper, we shall call the $\hat{z}$-direction 'vertical' while the $x$ - and $y$-directions, perpendicular to $\hat{z}$, are termed 'horizonal' (Wu \& Roberts 2008). We shall also adopt a frame of reference that is attached to the precessing channel and in which the boundaries of the channel are at rest (hereinafter referred to as the body or mantle frame). In the body frame of reference, we choose a Cartesian coordinate system in that the fluid cavity of the channel is defined by $0 \leqslant y \leqslant \Gamma d, 0 \leqslant z \leqslant d$ and $-\infty<x<\infty$. The four walls of the channel are $y=0$ located at one vertical wall (called 'outer sidewall'), $y=\Gamma d$ at the another vertical wall (called 'inner sidewall'), while $z=0$ at one horizonal wall (called 'bottom') and $z=d$ at the another horizontal wall (called 'top'). Moreover, the $x$-direction, which is parallel to the four walls, will be termed 'azimuthal'. The above terminology is introduced because of its correspondence with an annular channel under the small-gap approximation (Gilman 1973; Busse 2005). In the body frame of reference, the precessionally driven flow in an incompressible fluid is governed by the two equations (see, for example, Greenspan 1968)

$$
\begin{gathered}
\frac{\partial \boldsymbol{u}}{\partial t}+\boldsymbol{u} \cdot \nabla \boldsymbol{u}+2\left(\Omega \hat{z}+\boldsymbol{\Omega}_{p}\right) \times \boldsymbol{u}=-\frac{1}{\rho} \nabla p+\nu \nabla^{2} \boldsymbol{u}+\boldsymbol{r} \times\left[\boldsymbol{\Omega}_{p} \times(\Omega \hat{z})\right], \\
\nabla \cdot \boldsymbol{u}=0,
\end{gathered}
$$

where $t$ is time, $\rho$ is the fluid density, $\boldsymbol{\Omega}_{p}$ represents the precession vector which is fixed in space, $p$ is a reduced pressure and $\boldsymbol{r}$ is the position vector; $\boldsymbol{u}$ is the threedimensional velocity field $\boldsymbol{u}=\left(u_{x}, u_{y}, u_{z}\right)$ with the corresponding unit vectors $(\hat{\boldsymbol{x}}, \hat{\boldsymbol{y}}, \hat{z})$. The last term on the right-hand side of (2.1) is known as the Poincare forcing which drives precessional flows against viscous dissipation. In the Cartesian coordinates attached to the channel, the precession vector $\boldsymbol{\Omega}_{p}$ is time dependent and given by

$$
\boldsymbol{\Omega}_{p}=(\epsilon \Omega)(\hat{\boldsymbol{x}} \cos \Omega t-\hat{\boldsymbol{y}} \sin \Omega t),
$$

whose amplitude is $\left|\boldsymbol{\Omega}_{p}\right|=\epsilon \Omega$. We shall employ the depth $d$ as the length scale, $\Omega^{-1}$ as the unit of time and $\rho d^{2} \Omega^{2}$ as the unit of pressure, which lead to the dimensionless governing equations (Mason \& Kerswell 2002; Wu \& Roberts 2008):

$$
\begin{aligned}
\frac{\partial \boldsymbol{u}}{\partial t}+\boldsymbol{u} \cdot \nabla \boldsymbol{u}+2 \hat{z} \times \boldsymbol{u}+\nabla p= & E \nabla^{2} \boldsymbol{u}+2 \epsilon \boldsymbol{u} \times(\hat{\boldsymbol{x}} \cos t-\hat{\boldsymbol{y}} \sin t) \\
& +2 \epsilon z(\hat{\boldsymbol{x}} \cos t-\hat{\boldsymbol{y}} \sin t), \\
\nabla \cdot \boldsymbol{u}= & 0,
\end{aligned}
$$

where the Ekman number, $E=v / \Omega d^{2}$, provides the measure of relative importance between the typical viscous force and the Coriolis force, and the Poincare number $\epsilon=\left|\boldsymbol{\Omega}_{p}\right| / \Omega$ quantifies the strength of the precessional forcing (Wu \& Roberts 2008). In comparison with the pressure in (2.1), the pressure $p$ in (2.4) is modified by including a potential term from the Poincare forcing. We shall also introduce the Reynolds number Re (Meunier et al. 2008) defined as

$$
R e=\frac{(\epsilon \Omega) d^{2}}{v}=\frac{\epsilon}{E}
$$


In the body frame, the flow on the bounding surface of a precessing channel is at rest, which requires

$$
\hat{\boldsymbol{n}} \cdot \boldsymbol{u}=\hat{\boldsymbol{n}} \times \boldsymbol{u}=0
$$

on the four boundaries of a channel - the bottom at $z=0$, the top at $z=1$, the outer sidewall at $y=0$ and the inner sidewall at $y=\Gamma$ - where $\hat{\boldsymbol{n}}$ denotes the unit normal of the bounding surface. The problem defined by (2.4)-(2.5) subject to the boundary conditions (2.6) will be solved asymptotically for an arbitrarily small but fixed $E$ with small and moderate Reynolds numbers in $\S 3$ and numerically for both weakly and strongly precessing flows in $\S 4$.

\section{Asymptotic analysis}

When the Poincare number $\epsilon$ is sufficiently small at a fixed small $E$, we may assume that the higher-order terms in (2.4), $\boldsymbol{u} \cdot \nabla \boldsymbol{u}$ and $\epsilon \boldsymbol{u} \times(\hat{\boldsymbol{x}} \cos t-\hat{\boldsymbol{y}} \sin t)$, can be neglected, leading to

$$
\begin{gathered}
\frac{\partial \boldsymbol{u}}{\partial t}+2 \hat{z} \times \boldsymbol{u}+\nabla p=E \nabla^{2} \boldsymbol{u}+2 \epsilon z(\hat{\boldsymbol{x}} \cos t-\hat{\boldsymbol{y}} \sin t), \\
\nabla \cdot \boldsymbol{u}=0,
\end{gathered}
$$

solutions of which, as we shall demonstrate later, provide a satisfactory quantitative agreement with the fully nonlinear solutions in the range $0<R e \leqslant O\left(10^{2}\right)$ for the exactly resonant precessing flow and in the range $0<R e \leqslant O\left(10^{3}\right)$ for the nonresonant flow. At first glance, $-2 \epsilon z(\hat{\boldsymbol{x}} \sin t+\hat{\boldsymbol{y}} \cos t)$ satisfies (3.1) with the viscous term omitted and, hence, may represent the mainstream solution of the problem. However, it cannot be the mainstream solution because the inviscid boundary condition at the sidewalls is not satisfied.

In our asymptotic analysis, we postulate the following underlying physics and dynamics for a weakly precessing flow in a channel: the precessing flow can be approximated by either a single inviscid inertial mode or several inviscid inertial modes which is/are selected by a combined influence of the geometric factor $(\Gamma)$ and the spatial-temporal structure of the Poincare forcing, modified by viscous effects mainly via the viscous boundary layers on the four bounding surfaces and energetically driven by precession against viscous dissipation. It follows that our asymptotic analysis with the non-slip boundary condition will be based on three hypotheses: (i) the weakly precessing flow is oscillatory and axisymmetric $(\partial / \partial x=0)$ because the Poincare forcing, the final term in (2.4), is oscillatory and independent of the azimuthal variable $x$; (ii) the leading-order interior precessing flow can be represented by either a single inertial mode or by a combination of several inertial modes whose explicit analytical expressions in the inviscid limit are available; and (iii) there exist strong viscous boundary layers on the bounding surface of the channel that either control (in the case of resonant excitation) or modify (in the case of non-resonant excitation) the leading-order precessing flow. In connection with hypothesis (ii), an axisymmetric inertial mode in the inviscid limit is governed by the two equations

$$
\begin{gathered}
2 \mathrm{i} \sigma_{j n} \boldsymbol{u}_{j n}+2 \hat{z} \times \boldsymbol{u}_{j n}+\nabla p_{j n}=0, \\
\nabla \cdot \boldsymbol{u}_{j n}=0,
\end{gathered}
$$

where $\mathrm{i}=\sqrt{-1}$ and $\sigma_{j n}$ is the half-frequency of an inertial mode, subject to the condition of vanishing normal flow

$$
\hat{\boldsymbol{n}} \cdot \hat{\boldsymbol{u}}_{j n}=0
$$


at the bounding surface of the channel. Two integer numbers, $j$ and $n$, describe the radial and vertical structures of an inertial mode, respectively. The half-frequency of an axisymmetric inertial mode in (3.3), $\sigma_{j n}$, is given by

$$
\sigma_{j n}=\frac{n}{\sqrt{n^{2}+(j / \Gamma)^{2}}}, \quad \text { for } j=1,2, \ldots, \quad n= \pm 1, \pm 2, \ldots,
$$

satisfying the bound $0<\left|\sigma_{j n}\right|<1$ (see, for example, Liao \& Zhang 2009), while explicit solutions for the pressure $p_{j n}$ and the velocity $\boldsymbol{u}_{j n}$ can be written in the complex form

$$
\begin{aligned}
p_{j n}(y, z) & =\cos \left(\frac{j \pi y}{\Gamma}\right) \cos n \pi z, \\
\hat{\boldsymbol{x}} \cdot \boldsymbol{u}_{j n}(y, z) & =\frac{j \pi}{2 \Gamma\left(1-\sigma_{j n}^{2}\right)}\left[\sin \left(\frac{j \pi y}{\Gamma}\right) \cos n \pi z\right], \\
\hat{\boldsymbol{y}} \cdot \boldsymbol{u}_{j n}(y, z) & =\frac{\mathrm{i} \sigma_{j n} j \pi}{2 \Gamma\left(1-\sigma_{j n}^{2}\right)}\left[\sin \left(\frac{j \pi y}{\Gamma}\right) \cos n \pi z\right], \\
\hat{z} \cdot \boldsymbol{u}_{j n}(y, z) & =-\frac{\mathrm{i} n \pi}{2 \sigma_{j n}}\left[\cos \left(\frac{j \pi y}{\Gamma}\right) \sin n \pi z\right] .
\end{aligned}
$$

It is worth mentioning that the $x$-component of the inertial modes, given by (3.8), automatically satisfies the no-slip boundary condition at the sidewalls of the channel. In consequence, the viscous boundary-layer adjustments for this component at the sidewalls are not required, which simplifies the boundary-layer analysis. In (3.7)-(3.10), the aspect ratio $\Gamma$ represents a key parameter in controlling the primary character of precessionally driven flows. We shall concentrate on the regime of moderate aspect ratio with $E^{1 / 2} \ll \Gamma \leqslant O(1)$.

It is of primary importance to note that strong resonance takes place when the aspect ratio $\Gamma=\sqrt{3} j / 3 n$, (i.e. when $\sigma_{j n}=1 / 2$ ) for small values of the wavenumbers $n$ and $j$ at a fixed small $E$. Because of the vertical symmetry property of the Poincare forcing, however, the vertical wavenumber $n$ must be odd at resonance. For example, all the inertial modes with $n=2 K-1$ and $j=2 K-1, K=1,2,3, \ldots$, are resonant at $\Gamma=1 / \sqrt{3}$. In other words, it is always multiply resonant. However, the higher-order modes are viscously damped by a factor of $O\left(K^{2}\right)$ and, consequently, only the inertial modes with lowest orders in $j$ and $n$ are the most resonant and physically significant. It follows that as the aspect ratio $\Gamma$ varies at a fixed small $E$, precessionally driven flows may be divided into three different categories: (i) the double-inertial-mode resonance at $\Gamma=1 / \sqrt{3}$ where two principal inertial modes, $(j=1, n=1)$ and $(j=3, n=3)$, are significant at exact resonance with the Poincare forcing; (ii) the single-inertial-mode resonance at $\Gamma=\sqrt{3}$ where only a single inertial mode $(j=3, n=1)$ is significant at exact resonance, for which the higher modes like $(j=9, n=3)$ make a negligible contribution; and (iii) multiple-inertial-mode excitation at non-resonance when $|\Gamma-1 / \sqrt{3}| \gg E^{1 / 2}$ or $|\Gamma-\sqrt{3}| \gg E^{1 / 2}$, in which a large number of inertial modes may be excited by the Poincaré forcing. We shall not consider the weak resonance taking place when $\Gamma \gg 1$ with large values of $n$ and $j$. Evidently, the simplest asymptotic solution is offered by the single-mode resonance at $\Gamma=\sqrt{3}$, which will be discussed first.

\subsection{Single-inertial-mode resonance}

In a precessing channel with aspect ratio $\Gamma=\sqrt{3}$, an asymptotic solution $\boldsymbol{u}$ for a weakly precessing flow with $\epsilon \ll 1$ at a fixed small $E$ can be, following the three 
hypotheses, separated into a single-inertial-mode interior flow $\boldsymbol{u}_{31}$ and the corresponding boundary-layer correction $\widetilde{\boldsymbol{u}}_{31}$, together with a weak geostrophic flow $\boldsymbol{u}_{G}$. An asymptotic expansion for the velocity $\boldsymbol{u}$ and the reduced pressure $p$ takes the form

$$
\begin{aligned}
& \boldsymbol{u}(y, z, t)=\left[\left(\boldsymbol{u}_{G}+\widetilde{\boldsymbol{u}}_{G}\right)+\left(\mathscr{C}_{31} \boldsymbol{u}_{31}+\hat{\boldsymbol{u}}\right)+\widetilde{\boldsymbol{u}}_{31}\right] \mathrm{e}^{\mathrm{i} t}, \\
& p(y, z, t)=\left[\left(p_{G}+\widetilde{p}_{G}\right)+\left(\mathscr{C}_{31} p_{31}+\hat{p}\right)+\widetilde{p}_{31}\right] \mathrm{e}^{\mathrm{i} t},
\end{aligned}
$$

where $\mathscr{C}_{31}$ is an unknown complex number to be determined in the second-order problem, $\left|\boldsymbol{u}_{G}\right|=O(\epsilon)$ denotes the interior geostrophic flow while $\left|\widetilde{\boldsymbol{u}}_{G}\right|=O(\epsilon)$ corresponds to its boundary correction, the single inertial mode $\left(\boldsymbol{u}_{31}, p_{31}\right)$ for the interior precessing flow, given by (3.7)-(3.10) with $j=3$ and $n=1$ and with $\left|\mathscr{C}_{31} \boldsymbol{u}_{31}\right|=O\left(\epsilon / E^{1 / 2}\right)$, is corrected by the viscous boundary layer $\left(\widetilde{\boldsymbol{u}}_{31}, \widetilde{p}_{31}\right)$ with $\left|\widetilde{\boldsymbol{u}}_{31}\right|=O\left(\epsilon / E^{1 / 2}\right)$, which generates, through the boundary flux, the secondary interior flow, $(\hat{\boldsymbol{u}}, \hat{p})$, where $|\hat{\boldsymbol{u}}|=O(\epsilon)$. Note that the interior geostrophic flow, $\boldsymbol{u}_{G}$, is independent of $z$ (see, for example, Greenspan 1968).

Four sets of equations - describing the boundary-layer flow $\widetilde{\boldsymbol{u}}_{31}$, the secondary interior flow $\hat{\boldsymbol{u}}$, the interior geostrophic flow $\boldsymbol{u}_{G}$, the boundary geostrophic flow $\widetilde{\boldsymbol{u}}_{G}-$ can be derived after substituting the asymptotic expansions (3.11) and (3.12) into (3.1) and (3.2). First, the viscous boundary-layer flow $\widetilde{\boldsymbol{u}}_{31}$ is governed by

$$
\begin{aligned}
\mathrm{i} \widetilde{\boldsymbol{u}}_{31}+2 \hat{z} \times \widetilde{\boldsymbol{u}}_{31}+\hat{\boldsymbol{n}} \cdot \nabla \widetilde{p}_{31} & =\frac{\partial^{2} \widetilde{\boldsymbol{u}}_{31}}{\partial \xi^{2},} \\
\hat{\boldsymbol{n}} \cdot \nabla \times\left(\hat{\boldsymbol{n}} \times \widetilde{\boldsymbol{u}}_{31}\right) & =\frac{\partial\left(\hat{\boldsymbol{n}} \cdot \widetilde{\boldsymbol{u}}_{31}\right)}{\partial \xi},
\end{aligned}
$$

subject to the boundary condition

$$
\hat{\boldsymbol{n}} \times\left(\widetilde{\boldsymbol{u}}_{31}+\mathscr{C}_{31} \boldsymbol{u}_{31}\right)=0,
$$

which ensures that the tangential velocity vanishes at the bounding surface of the container. In (3.13) and (3.14), $\xi$ denotes the stretched boundary-layer variable: $\xi=z E^{-1 / 2}$ on the bottom at $z=0$ (which will be referred to as $S_{1}$ ), $\xi=(1-z) E^{-1 / 2}$ on the top at $z=1$ (referred to as $S_{2}$ ), $\xi=y E^{-1 / 2}$ for the boundary layer on the outer sidewall at $y=0$ (referred to as $S_{3}$ ) and $\xi=(\Gamma-y) E^{-1 / 2}$ on the inner sidewall at $y=\Gamma$ (referred to as $\left.S_{4}\right)$. Second, the secondary interior flow is described by

$$
\begin{gathered}
\mathrm{i} \hat{\boldsymbol{u}}+2 \hat{z} \times \hat{\boldsymbol{u}}+\nabla \hat{p}=E \mathscr{C}_{31} \nabla^{2} \boldsymbol{u}_{31}+2 \epsilon z(\hat{\boldsymbol{x}}+\mathrm{i} \hat{\boldsymbol{y}}), \\
\nabla \cdot \hat{\boldsymbol{u}}=0,
\end{gathered}
$$

where the term $E \nabla^{2} \boldsymbol{u}_{31}$ represents the interior viscous dissipation, which is retained because it can be readily incorporated and may make a significant contribution (Zhang \& Liao 2008). Linking the boundary-layer flow $\widetilde{\boldsymbol{u}}_{31}$ to the secondary interior flow $\hat{\boldsymbol{u}}$ is the normal component of the mass flux at the edge of the viscous boundary layer

$$
\hat{\boldsymbol{n}} \cdot \hat{\boldsymbol{u}}=E^{1 / 2} \int_{0}^{\infty} \hat{\boldsymbol{n}} \cdot \nabla \times\left(\hat{\boldsymbol{n}} \times \widetilde{\boldsymbol{u}}_{31}\right) \mathrm{d} \xi,
$$

providing the asymptotic matching condition required in evaluating the solvability condition for (3.16)-(3.17) which can be obtained by multiplying (3.16) with the complex conjugate of $\boldsymbol{u}_{31}$ and then integrating the resulting equation over the channel. Third, an equation for the interior geostrophic flow $\boldsymbol{u}_{G}$ can be derived by inserting (3.11) and (3.12) into (3.1) and then integrating the azimuthal component of the 
resulting equation:

$$
\int_{0}^{1} \mathrm{i} \hat{\boldsymbol{x}} \cdot \boldsymbol{u}_{G}(y) \mathrm{d} z=2 \epsilon \int_{0}^{1} z \hat{\boldsymbol{x}} \cdot(\hat{\boldsymbol{x}}+\mathrm{i} \hat{\boldsymbol{y}}) \mathrm{d} z .
$$

Finally, the viscous correction for the geostrophic flow $\boldsymbol{u}_{G}$ is governed by

$$
\begin{aligned}
\mathrm{i} \widetilde{\boldsymbol{u}}_{G}+2 \hat{z} \times \widetilde{\boldsymbol{u}}_{G}+\hat{\boldsymbol{n}} \cdot \nabla \widetilde{p}_{G} & =\frac{\partial^{2} \widetilde{\boldsymbol{u}}_{G}}{\partial \xi^{2},} \\
\hat{\boldsymbol{n}} \cdot \nabla \times\left(\hat{\boldsymbol{n}} \times \widetilde{\boldsymbol{u}}_{G}\right) & =\frac{\partial\left(\hat{\boldsymbol{n}} \cdot \widetilde{\boldsymbol{u}}_{G}\right)}{\partial \xi},
\end{aligned}
$$

subject to the boundary condition

$$
\hat{\boldsymbol{n}} \times\left(\widetilde{\boldsymbol{u}}_{G}+\boldsymbol{u}_{G}\right)=0 .
$$

The analysis of the geostrophic boundary flow is entirely analogous to that for $\widetilde{\boldsymbol{u}}_{31}$ and, consequently, will not be discussed in detail.

On solving the boundary-layer equations (3.13) and (3.14) for $\widetilde{\boldsymbol{u}}_{31}$ on the four bounding walls of the channel, the solvability condition for (3.16) and (3.17), in conjunction with the mass flux from the four viscous boundary layers, yields an equation which determines the unknown complex coefficient $\mathscr{C}_{31}$ :

$$
\begin{aligned}
\frac{8 \epsilon}{\pi^{2}}= & -E_{\mathscr{C}_{31}}\left(\frac{8 \pi^{4} \sqrt{3}}{3}\right) \\
& +E^{1 / 2}\left\{\int_{S_{1}}\left[\left(\mathrm{i} \boldsymbol{u}_{31}^{*}-2 \hat{z} \times \boldsymbol{u}_{31}^{*}\right)_{z=0} \cdot\left(\int_{0}^{\infty} \widetilde{\boldsymbol{u}}_{31} \mathrm{~d} \xi\right)\right] \mathrm{d} S\right. \\
& +\int_{S_{2}}\left[\left(\mathrm{i} \boldsymbol{u}_{31}^{*}-2 \hat{z} \times \boldsymbol{u}_{31}^{*}\right)_{z=1} \cdot\left(\int_{0}^{\infty} \widetilde{\boldsymbol{u}}_{31} \mathrm{~d} \xi\right)\right] \mathrm{d} S \\
& +\int_{S_{3}}\left[\left(\mathrm{i} \boldsymbol{u}_{31}^{*}-2 \hat{z} \times \boldsymbol{u}_{31}^{*}\right)_{y=0} \cdot\left(\int_{0}^{\infty} \widetilde{\boldsymbol{u}}_{31} \mathrm{~d} \xi\right)\right] \mathrm{d} S \\
& \left.+\int_{S_{4}}\left[\left(\mathrm{i} \boldsymbol{u}_{31}^{*}-2 \hat{z} \times \boldsymbol{u}_{31}^{*}\right)_{y=\Gamma} \cdot\left(\int_{0}^{\infty} \widetilde{\boldsymbol{u}}_{31} \mathrm{~d} \xi\right)\right] \mathrm{d} S\right\},
\end{aligned}
$$

where $\boldsymbol{u}_{31}^{*}$ denotes the complex conjugate of $\boldsymbol{u}_{31}$ and $\int_{S_{j}} f \mathrm{~d} S$ means the surface integration with $f$ evaluated on the bounding wall $S_{j}$. The surface integrations in (3.23) are derived from the product of $p_{31}^{*}$, the complex conjugate of $p_{31}$, and the boundary mass flux. With the explicit expressions for $\widetilde{\boldsymbol{u}}_{31}$ on the four bounding surfaces, $S_{j}, j=1,2,3,4$, of the channel, which are given in (3.24), we can readily evaluate the four integrations in (3.23) and, hence, determine the value of $\mathscr{C}_{31}$ analytically.

An analytical expression for the weakly precessing flow at exact resonance satisfying the non-slip boundary condition is then obtained by inserting $\mathscr{C}_{31}, \widetilde{\boldsymbol{u}}_{31}$ and $\boldsymbol{u}_{31}$ into the asymptotic expansion (3.11):

$$
\begin{aligned}
\boldsymbol{u}= & \left(\boldsymbol{u}_{G}+\widetilde{\boldsymbol{u}}_{G}\right)-\frac{96(\epsilon / \sqrt{E}) \mathrm{e}^{\mathrm{i} t}}{\pi^{4}\left\{9 \sqrt{2}[(1+\sqrt{3})+(1-\sqrt{3}) \mathrm{i}]+32 \sqrt{3} \pi^{2} \sqrt{E}\right\}} \\
& \times\left\{( \frac { \pi \operatorname { s i n } \sqrt { 3 } \pi y } { 2 \sqrt { 3 } } ) \left\{\left[4 \cos \pi z-\exp \left(-\frac{\sqrt{6}(1+\mathrm{i}) z}{2 \sqrt{E}}\right)-3 \exp \left(-\frac{\sqrt{2}(1-\mathrm{i}) z}{2 \sqrt{E}}\right)\right.\right.\right.
\end{aligned}
$$




$$
\begin{aligned}
& \left.+\exp \left(-\frac{\sqrt{6}(1+\mathrm{i})(1-z)}{2 \sqrt{E}}\right)+3 \exp \left(-\frac{\sqrt{2}(1-\mathrm{i})(1-z)}{2 \sqrt{E}}\right)\right] \hat{\boldsymbol{x}} \\
& +\mathrm{i}\left[2 \cos \pi z+\exp \left(-\frac{\sqrt{6}(1+\mathrm{i}) z}{2 \sqrt{E}}\right)-3 \exp \left(-\frac{\sqrt{2}(1-\mathrm{i}) z}{2 \sqrt{E}}\right)\right. \\
& \left.\left.-\exp \left(-\frac{\sqrt{6}(1+\mathrm{i})(1-z)}{2 \sqrt{E}}\right)+3 \exp \left(-\frac{\sqrt{2}(1-\mathrm{i})(1-z)}{2 \sqrt{E}}\right)\right] \hat{\boldsymbol{y}}\right\}-\mathrm{i} \pi \sin \pi z \\
& \left.\times\left[\cos \sqrt{3} \pi y-\exp \left(-\frac{\sqrt{2}(1+\mathrm{i}) y}{2 \sqrt{E}}\right)+\exp \left(-\frac{\sqrt{2}(1+\mathrm{i})(\Gamma-y)}{2 \sqrt{E}}\right)\right] \hat{z}\right\},
\end{aligned}
$$

which is valid for moderate values of the Reynolds number $R e$ for $\Gamma=\sqrt{3}$. Note that the real part of (3.24) represents the required asymptotic solution for the precessing flow. It is also worth noting that the amplitude of the vertical component in (3.24) is comparable with that of the horizontal components.

In the asymptotic solution (3.24), the oscillatory geostrophic flow satisfying the nonslip boundary condition can be derived by a similar procedure. The leading-order expression takes the form

$$
\begin{aligned}
\boldsymbol{u}_{G}+\widetilde{\boldsymbol{u}}_{G}= & \frac{\mathrm{i} \epsilon}{2}\left\{\left[-2+\exp \left(-\frac{\sqrt{6}(1+\mathrm{i}) z}{2 \sqrt{E}}\right)+\exp \left(-\frac{\sqrt{2}(1-\mathrm{i}) z}{2 \sqrt{E}}\right)\right.\right. \\
& \left.+\exp \left(-\frac{\sqrt{6}(1+\mathrm{i})(1-z)}{2 \sqrt{E}}\right)+\exp \left(-\frac{\sqrt{2}(1-\mathrm{i})(1-z)}{2 \sqrt{E}}\right)\right] \hat{\boldsymbol{x}} \\
& +\mathrm{i}\left[-\exp \left(-\frac{\sqrt{6}(1+\mathrm{i}) z}{2 \sqrt{E}}\right)+\exp \left(-\frac{\sqrt{2}(1-\mathrm{i}) z}{2 \sqrt{E}}\right)\right. \\
& \left.-\exp \left(-\frac{\sqrt{6}(1+\mathrm{i})(1-z)}{2 \sqrt{E}}\right)+\exp \left(-\frac{\sqrt{2}(1-\mathrm{i})(1-z)}{2 \sqrt{E}}\right)\right] \hat{\boldsymbol{y}} \\
& \left.+2\left[\exp \left(-\frac{\sqrt{2}(1+\mathrm{i}) y}{2 \sqrt{E}}\right)+\exp \left(-\frac{\sqrt{2}(1-\mathrm{i})(\Gamma-y)}{2 \sqrt{E}}\right)\right] \hat{\boldsymbol{x}}\right\} \mathrm{e}^{\mathrm{i} t},
\end{aligned}
$$

which is valid for any value of the aspect ratio $\Gamma$. Note that although the amplitude of the geostrophic flow is much smaller than that of the principal inertial mode at exact resonance, it is comparable to that of excitable inertial modes at nonresonance. Moreover, the geostrophic flow may play a critical role in the nonlinear interaction (see, for example, Kobine 1995; Kerswell 2002) leading to the transition to a three-dimensional chaotic flow at large Reynolds numbers. To measure the strength of the resonant precessing flow, we introduce the averaged kinetic energy, $E_{k i n}$, defined as

$$
E_{k i n}=\frac{1}{2 \pi} \int_{0}^{2 \pi} \frac{1}{2 V}\left[\int_{V}|\operatorname{Re}(\boldsymbol{u})|^{2} \mathrm{~d} V\right] \mathrm{d} t,
$$



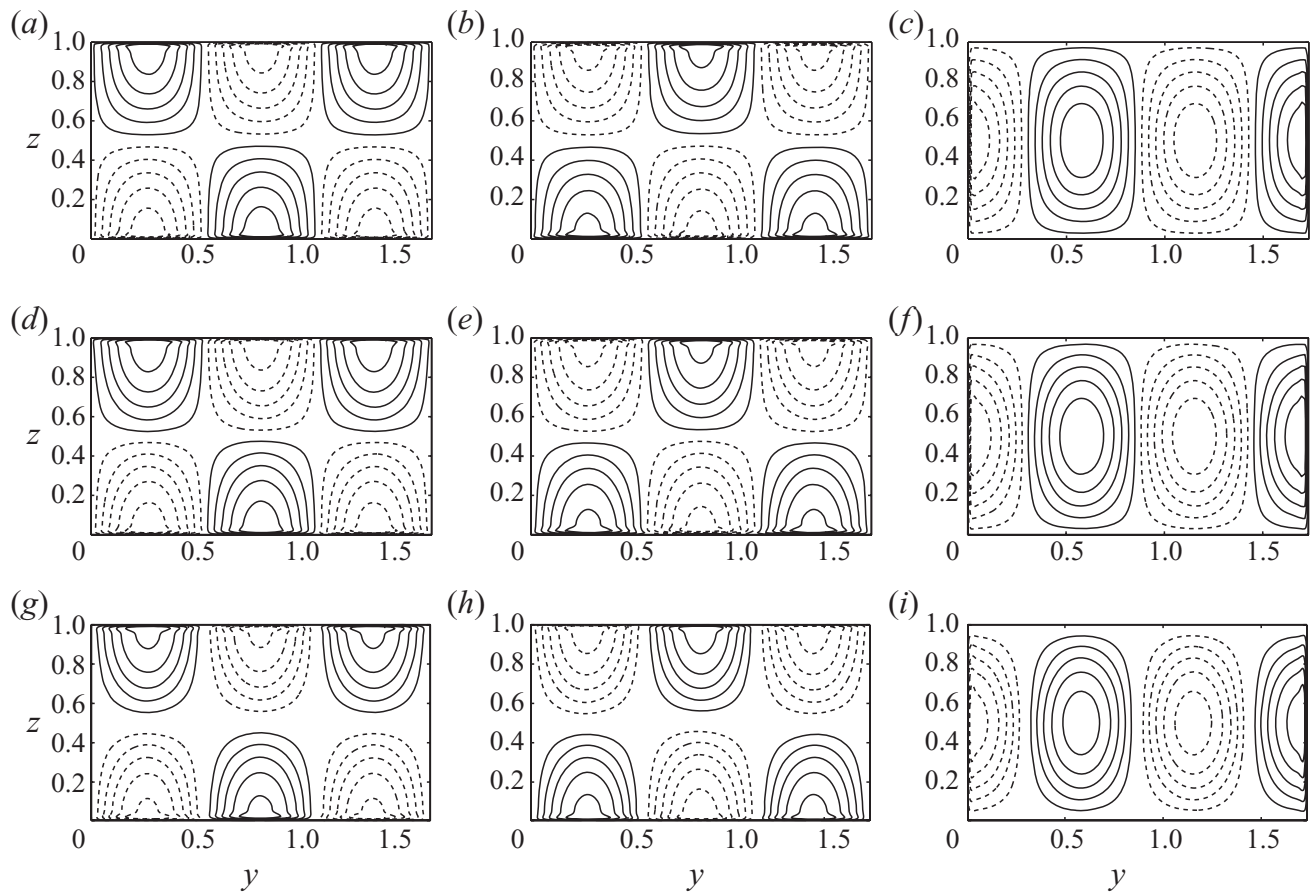

Figure 1. Contours of $(a) u_{x},(b) u_{y}$ and (c) $u_{z}$ (top) in a vertical $y z$ plane for the asymptotic solution computed from the analytical expression (3.24); contours of $(d) u_{x},(e) u_{y}$ and $(f) u_{z}$ (middle) computed from the two-dimensional numerical analysis; contours of $(g) u_{x}$, (h) $u_{y}$ and $(i) u_{z}$ (bottom) from direct three-dimensional numerical simulation. The parameters for all three solutions are $\epsilon=5 \times 10^{-4}$ and $\Gamma=\sqrt{3}$ at $E=5 \times 10^{-5}$.

where $V$ denotes the volume of the channel. Using the analytical expressions (3.24) and (3.25), we obtain

$$
E_{k i n}=\frac{1}{4}\left[\frac{6144(\epsilon / \sqrt{E})^{2}}{\left.\pi^{6}\left\{9 \sqrt{2}[(1+\sqrt{3})]+32 \sqrt{3} \pi^{2} \sqrt{E}\right\}^{2}+162(1-\sqrt{3})^{2}\right]}+\epsilon^{2}\right],
$$

where the small contribution from the viscous boundary layers is not included. The asymptotic solution represented by the analytical expressions (3.24) and (3.27) will be compared directly to the result of two-dimensional and three-dimensional numerical simulations.

The typical structure of the weakly precessing flow satisfying the non-slip boundary condition, computed directly from the analytical expression (3.24), is illustrated in figure $1(a-c)$ for $\epsilon=5 \times 10^{-4}, \Gamma=\sqrt{3}$ and $E=5 \times 10^{-5}$. Also displayed in figure 1, for the purpose of easy comparison, are the two-dimensional numerical solution (figure $1 d-f$ ) and the fully three-dimensional nonlinear simulation (figure $1 g-$ i) obtained at exactly the same parameters. Evidently, there are no noticeable differences among the three solutions obtained via three fundamentally different methods. Furthermore, the kinetic energy $E_{k i n}$ computed from the expression (3.27) for $\epsilon=5.0 \times 10^{-4}$ and $\Gamma=\sqrt{3}$ is $E_{k i n}=5.11 \times 10^{-6}$ while the two-dimensional numerical analysis yields $E_{\text {kin }}=4.95 \times 10^{-6}$ and our direct three-dimensional nonlinear simulation gives $E_{\text {kin }}=4.47 \times 10^{-6}$ (the details of the numerics will be discussed in 
$\S 4)$. For a larger Poincaré number $\epsilon=10^{-3}$, the asymptotic expression (3.27) gives rise to $E_{\text {kin }}=2.05 \times 10^{-5}$ for $E=5 \times 10^{-5}$ while the corresponding two-dimensional numerics yields $E_{\text {kin }}=1.98 \times 10^{-5}$ and the three-dimensional nonlinear simulation gives $E_{k i n}=1.80 \times 10^{-5}$.

A satisfactory quantitative agreement between the asymptotic analysis and the two-dimensional and three-dimensional numerical simulation is achieved for small and moderate Reynolds numbers. This not only validates the asymptotic analysis but also confirms the crucial role played by viscosity at exact resonance. A comparison between the asymptotic and numerical results also indicates that the asymptotic solution (3.24) offers a reasonably good approximation to the fully nonlinear threedimensional simulation in the range $0<E^{1 / 2} R e<O(1)$, which will be discussed further.

\subsection{Double-inertial-mode resonance}

Consider now a precessing channel with aspect ratio $\Gamma=1 / \sqrt{3}$ which is also at exact resonance with the Poincare forcing. In this case, while the principal inertial mode $\boldsymbol{u}_{11}$ is predominant, the secondary resonant mode $\boldsymbol{u}_{33}$ makes a substantial contribution and, hence, should be included. An asymptotic solution $\boldsymbol{u}$ describing a weakly precessing flow for $E \ll 1$ and $\Gamma=1 / \sqrt{3}$ can be written in the form

$$
\begin{aligned}
& \boldsymbol{u}(y, z, t)=\left[\left(\boldsymbol{u}_{G}+\widetilde{\boldsymbol{u}}_{G}\right)+\left(\mathscr{C}_{11} \boldsymbol{u}_{11}+\mathscr{C}_{33} \boldsymbol{u}_{33}\right)+\hat{\boldsymbol{u}}+\widetilde{\boldsymbol{u}}\right] \mathrm{e}^{\mathrm{i} t}, \\
& p(y, z, t)=\left[\left(p_{G}+\widetilde{p}_{G}\right)+\left(\mathscr{C}_{11} p_{11}+\mathscr{C}_{33} p_{33}\right)+\hat{p}+\widetilde{p}\right] \mathrm{e}^{\mathrm{i} t},
\end{aligned}
$$

where $\mathscr{C}_{11}$ and $\mathscr{C}_{33}$ are complex coefficients to be determined, the two inviscid resonant inertial modes, $\left(\boldsymbol{u}_{11}, p_{11}\right)$ and $\left(\boldsymbol{u}_{33}, p_{33}\right)$, whose expressions are given by (3.7)-(3.10), must be corrected by the viscous boundary layer $\widetilde{\boldsymbol{u}}$ on the four bounding surfaces of the channel, and the secondary interior flow, $[\hat{\boldsymbol{u}}, \hat{p}]$, where $|\hat{\boldsymbol{u}}| \ll\left|\mathscr{C}_{11} \boldsymbol{u}_{11}+\mathscr{C}_{33} \boldsymbol{u}_{33}\right|$, is induced by the viscous effects. The expression for the geostrophic flow, $\boldsymbol{u}_{G}$ and $\widetilde{\boldsymbol{u}}_{G}$ given by (3.25), remains unchanged. Because the asymptotic analysis for the doublemode resonance is largely analogous to that of the single-mode analysis, we shall keep its discussion brief.

Upon inserting the asymptotic expansions (3.28) and (3.29) into (3.1) and (3.2), we can derive two equations governing the viscous boundary layer,

$$
\begin{aligned}
\mathrm{i} \widetilde{\boldsymbol{u}}+2 \hat{z} \times \widetilde{\boldsymbol{u}}+\hat{\boldsymbol{n}} \cdot \nabla \widetilde{p} & =\frac{\partial^{2} \widetilde{\boldsymbol{u}}}{\partial \xi^{2}}, \\
\hat{\boldsymbol{n}} \cdot \nabla \times(\hat{\boldsymbol{n}} \times \widetilde{\boldsymbol{u}}) & =\frac{\partial(\hat{\boldsymbol{n}} \cdot \widetilde{\boldsymbol{u}})}{\partial \xi},
\end{aligned}
$$

subject to the boundary condition

$$
\hat{\boldsymbol{n}} \times\left(\widetilde{\boldsymbol{u}}+\mathscr{C}_{11} \boldsymbol{u}_{11}+\mathscr{C}_{33} \boldsymbol{u}_{33}\right)=0
$$

on the bounding surface of the channel. The secondary interior flow $[\hat{\boldsymbol{u}}, \hat{p}]$ is governed by

$$
\begin{gathered}
\mathrm{i} \hat{\boldsymbol{u}}+2 \hat{z} \times \hat{\boldsymbol{u}}+\nabla \hat{p}=E\left(\mathscr{C}_{11} \nabla^{2} \boldsymbol{u}_{11}+\mathscr{C}_{33} \nabla^{2} \boldsymbol{u}_{33}\right)+2 \epsilon z(\hat{\boldsymbol{x}}+\mathrm{i} \hat{\boldsymbol{y}}), \\
\nabla \cdot \hat{\boldsymbol{u}}=0,
\end{gathered}
$$

subject to the boundary condition

$$
\hat{\boldsymbol{n}} \cdot \hat{\boldsymbol{u}}=E^{1 / 2} \int_{0}^{\infty} \hat{\boldsymbol{n}} \cdot \nabla \times(\hat{\boldsymbol{n}} \times \widetilde{\boldsymbol{u}}) \mathrm{d} \xi
$$


at the edge of the viscous boundary layers. The solvability condition for the inhomogeneous partial differential equation (3.33) subject to the normal flux condition on the four rigid walls gives rise to two complex equations for determining $\mathscr{C}_{11}$ and $\mathscr{C}_{33}$ :

$$
\begin{aligned}
\frac{8 \epsilon}{\pi^{2}}= & -E_{\mathscr{C}_{11}}\left(\frac{8 \pi^{4} \sqrt{3}}{9}\right) \\
& +E^{1 / 2}\left\{\int_{S_{1}}\left[\left(\mathrm{i} \boldsymbol{u}_{11}^{*}-2 \hat{z} \times \boldsymbol{u}_{11}^{*}\right)_{z=0} \cdot\left(\int_{0}^{\infty} \widetilde{\boldsymbol{u}} \mathrm{d} \xi\right)\right] \mathrm{d} S\right. \\
& +\int_{S_{2}}\left[\left(\mathrm{i} \boldsymbol{u}_{11}^{*}-2 \hat{z} \times \boldsymbol{u}_{11}^{*}\right)_{z=1} \cdot\left(\int_{0}^{\infty} \widetilde{\boldsymbol{u}} \mathrm{d} \xi\right)\right] \mathrm{d} S \\
& +\int_{S_{3}}\left[\left(\mathrm{i} \boldsymbol{u}_{11}^{*}-2 \hat{z} \times \boldsymbol{u}_{11}^{*}\right)_{y=0} \cdot\left(\int_{0}^{\infty} \widetilde{\boldsymbol{u}} \mathrm{d} \xi\right)\right] \mathrm{d} S \\
& \left.+\int_{S_{4}}\left[\left(\mathrm{i} \boldsymbol{u}_{11}^{*}-2 \hat{z} \times \boldsymbol{u}_{11}^{*}\right)_{y=\Gamma} \cdot\left(\int_{0}^{\infty} \widetilde{\boldsymbol{u}} \mathrm{d} \xi\right)\right] \mathrm{d} S\right\}
\end{aligned}
$$

and

$$
\begin{aligned}
\frac{8 \epsilon}{9 \pi^{2}}= & -E_{\mathscr{C}_{33}}\left(72 \pi^{4} \sqrt{3}\right) \\
& +E^{1 / 2}\left\{\int_{S_{1}}\left[\left(\mathrm{i} \boldsymbol{u}_{33}^{*}-2 \hat{z} \times \boldsymbol{u}_{33}^{*}\right)_{z=0} \cdot\left(\int_{0}^{\infty} \widetilde{\boldsymbol{u}} \mathrm{d} \xi\right)\right] \mathrm{d} S\right. \\
& +\int_{S_{2}}\left[\left(\mathrm{i} \boldsymbol{u}_{33}^{*}-2 \hat{z} \times \boldsymbol{u}_{33}^{*}\right)_{z=1} \cdot\left(\int_{0}^{\infty} \widetilde{\boldsymbol{u}} \mathrm{d} \xi\right)\right] \mathrm{d} S \\
& +\int_{S_{3}}\left[\left(\mathrm{i} \boldsymbol{u}_{33}^{*}-2 \hat{z} \times \boldsymbol{u}_{33}^{*}\right)_{y=0} \cdot\left(\int_{0}^{\infty} \widetilde{\boldsymbol{u}} \mathrm{d} \xi\right)\right] \mathrm{d} S \\
& \left.+\int_{S_{4}}\left[\left(i \boldsymbol{u}_{33}^{*}-2 \hat{z} \times \boldsymbol{u}_{33}^{*}\right)_{y=\Gamma} \cdot\left(\int_{0}^{\infty} \widetilde{\boldsymbol{u}} \mathrm{d} \xi\right)\right] \mathrm{d} S\right\} .
\end{aligned}
$$

It is worth noting that $\mathscr{C}_{11}$ and $\mathscr{C}_{33}$, in the double resonant case, are not coupled by the effect of the viscous boundary layers and, hence, can be readily obtained after solving (3.30) and (3.31) for the boundary flow $\widetilde{\boldsymbol{u}}$, and then performing the required integration in (3.36) and (3.37). The substitution of $\mathscr{C}_{11}$ and $\mathscr{C}_{33}$ into the expansion yields an analytical expression for the precessing flow at the double-mode resonance at $\Gamma=1 / \sqrt{3}$ :

$$
\boldsymbol{u}=\left(\boldsymbol{u}_{G}+\widetilde{\boldsymbol{u}}_{G}\right)+\boldsymbol{V}_{11}+\boldsymbol{V}_{33},
$$

where the geostrophic flow $\left(\boldsymbol{u}_{G}+\widetilde{\boldsymbol{u}}_{G}\right)$ is still given by (3.25) while $\boldsymbol{V}_{11}$, in association with the primary resonant mode $\boldsymbol{u}_{11}$, takes the form

$$
\begin{aligned}
\boldsymbol{V}_{11}= & -\frac{288(\epsilon / \sqrt{E}) \mathrm{e}^{\mathrm{i} t}}{\pi^{4}\left\{3 \sqrt{2}[(7+3 \sqrt{3})+(7-3 \sqrt{3}) \mathrm{i}]+32 \sqrt{3} \pi^{2} \sqrt{E}\right\}} \\
& \times\left\{( \frac { \pi \operatorname { s i n } \sqrt { 3 } \pi y } { 2 \sqrt { 3 } } ) \left\{\left[4 \cos \pi z-\exp \left(-\frac{\sqrt{6}(1+\mathrm{i}) z}{2 \sqrt{E}}\right)-3 \exp \left(-\frac{\sqrt{2}(1-\mathrm{i}) z}{2 \sqrt{E}}\right)\right.\right.\right. \\
& \left.+\exp \left(-\frac{\sqrt{6}(1+\mathrm{i})(1-z)}{2 \sqrt{E}}\right)+3 \exp \left(-\frac{\sqrt{2}(1-\mathrm{i})(1-z)}{2 \sqrt{E}}\right)\right] \hat{\boldsymbol{x}}
\end{aligned}
$$




$$
\begin{aligned}
& +\mathrm{i}\left[2 \cos \pi z+\exp \left(-\frac{\sqrt{6}(1+\mathrm{i}) z}{2 \sqrt{E}}\right)-3 \exp \left(-\frac{\sqrt{2}(1-\mathrm{i}) z}{2 \sqrt{E}}\right)\right. \\
& \left.\left.-\exp \left(-\frac{\sqrt{6}(1+\mathrm{i})(1-z)}{2 \sqrt{E}}\right)+3 \exp \left(-\frac{\sqrt{2}(1-\mathrm{i})(1-z)}{2 \sqrt{E}}\right)\right] \hat{\boldsymbol{y}}\right\}-\mathrm{i} \pi \sin \pi z \\
& \left.\times\left[\cos \sqrt{3} \pi y-\exp \left(-\frac{\sqrt{2}(1+\mathrm{i}) y}{2 \sqrt{E}}\right)+\exp \left(-\frac{\sqrt{2}(1+\mathrm{i})(\Gamma-y)}{2 \sqrt{E}}\right)\right] \hat{z}\right\},
\end{aligned}
$$

and $\boldsymbol{V}_{33}$, in association with the secondary resonant mode $\boldsymbol{u}_{33}$, is given by

$$
\begin{aligned}
\boldsymbol{V}_{33}= & -\frac{288(\epsilon / \sqrt{E}) \mathrm{e}^{\mathrm{i} t}}{\pi^{4}\left\{3 \sqrt{2}[(7+3 \sqrt{3})+(7-3 \sqrt{3}) \mathrm{i}]+288 \sqrt{3} \pi^{2} \sqrt{E}\right\}} \\
& \times\left\{( \frac { \pi \operatorname { s i n } 3 \sqrt { 3 } \pi y } { 5 4 \sqrt { 3 } } ) \left\{\left[4 \cos 3 \pi z-\exp \left(-\frac{\sqrt{6}(1+\mathrm{i}) z}{2 \sqrt{E}}\right)-3 \exp \left(-\frac{\sqrt{2}(1-\mathrm{i}) z}{2 \sqrt{E}}\right)\right.\right.\right. \\
& \left.+\exp \left(-\frac{\sqrt{6}(1+\mathrm{i})(1-z)}{2 \sqrt{E}}\right)+3 \exp \left(-\frac{\sqrt{2}(1-\mathrm{i})(1-z)}{2 \sqrt{E}}\right)\right] \hat{\boldsymbol{x}} \\
& +\mathrm{i}\left[2 \cos 3 \pi z+\exp \left(-\frac{\sqrt{6}(1+\mathrm{i}) z}{2 \sqrt{E}}\right)-3 \exp \left(-\frac{\sqrt{2}(1-\mathrm{i}) z}{2 \sqrt{E}}\right)\right. \\
& \left.\left.-\exp \left(-\frac{\sqrt{6}(1+\mathrm{i})(1-z)}{2 \sqrt{E}}\right)+3 \exp \left(-\frac{\sqrt{2}(1-\mathrm{i})(1-z)}{2 \sqrt{E}}\right)\right] \hat{\boldsymbol{y}}\right\}-\mathrm{i} \frac{\pi}{27} \sin 3 \pi z \\
& \left.\times\left[\cos 3 \sqrt{3} \pi y-\exp \left(-\frac{\sqrt{2}(1+\mathrm{i}) y}{2 \sqrt{E}}\right)+\exp \left(-\frac{\sqrt{2}(1+\mathrm{i})(\Gamma-y)}{2 \sqrt{E}}\right)\right] \hat{z}\right\} .
\end{aligned}
$$

Note that the secondary resonant mode, $\boldsymbol{u}_{33}$, makes about $5 \%$ contribution towards the amplitude of the precessing flow. The total kinetic energy of the flow including the geostrophic flow is given by

$$
\begin{aligned}
E_{k i n}= & \frac{512(\epsilon / \sqrt{E})^{2}}{\pi^{6}}\left\{\frac{27}{\left[3 \sqrt{2}(7+3 \sqrt{3})+32 \pi^{2} \sqrt{3 E}\right]^{2}+18(7-3 \sqrt{3})^{2}}\right. \\
& \left.+\frac{1}{27\left[3 \sqrt{2}(7+3 \sqrt{3})+288 \pi^{2} \sqrt{3 E}\right]^{2}+486(7-3 \sqrt{3})^{2}}\right\}+\frac{\epsilon^{2}}{4},
\end{aligned}
$$

where the small contribution from the viscous boundary layers is excluded.

The analytical expressions (3.38) and (3.41) are compared directly to the two-dimensional numerical analysis and fully three-dimensional direct numerical simulations. The spatial structure of the precessing flow for $\Gamma=1 / \sqrt{3}$, computed from expression (3.38), is displayed in figure $2(a-c)$ for $\epsilon=5 \times 10^{-4}$ and $E=5 \times 10^{-5}$. Also displayed in the figure are the two-dimensional numerical solution (figure $2 d-$ $f$ ) and the direct three-dimensional nonlinear simulation (figure $2 g-i$ ) obtained at exactly the same parameters. There are no significant differences among the 
(a)

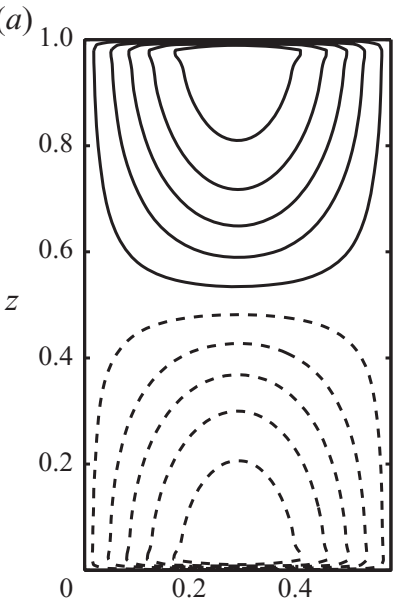

(d)

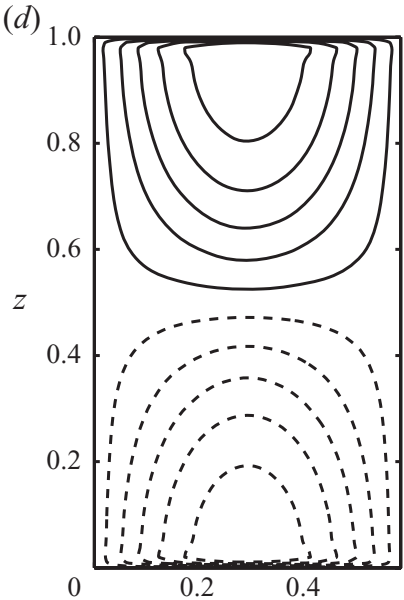

$(g)$

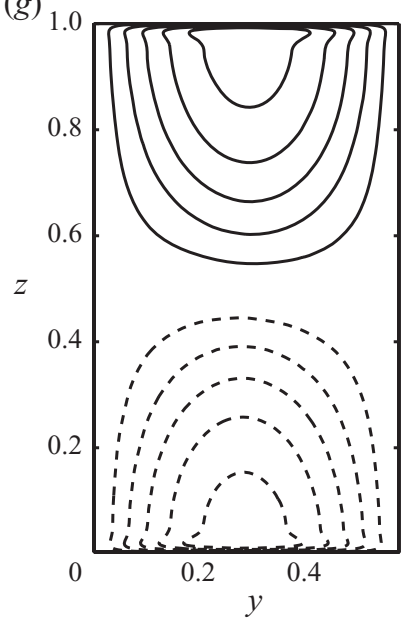

(b)

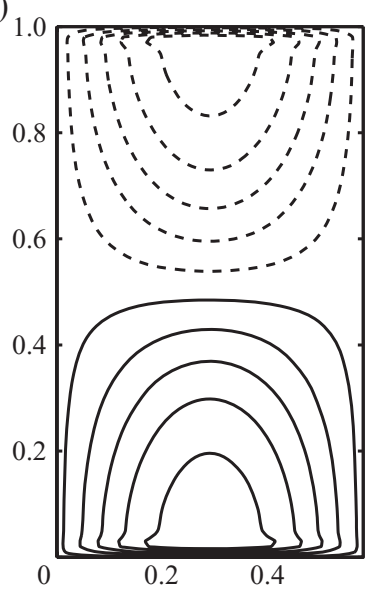

$(e)$

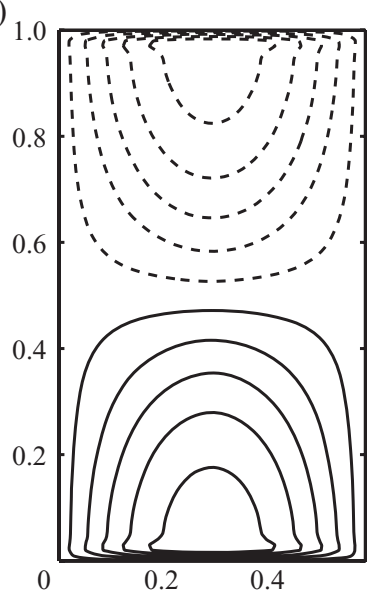

(h)

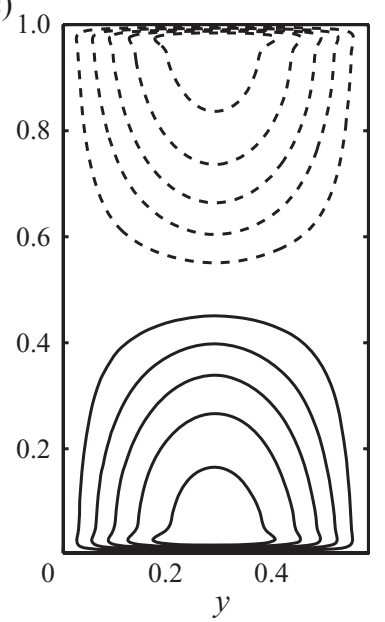

(c)

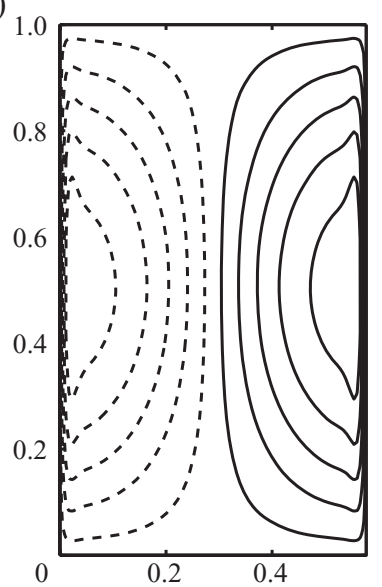

$(f)$

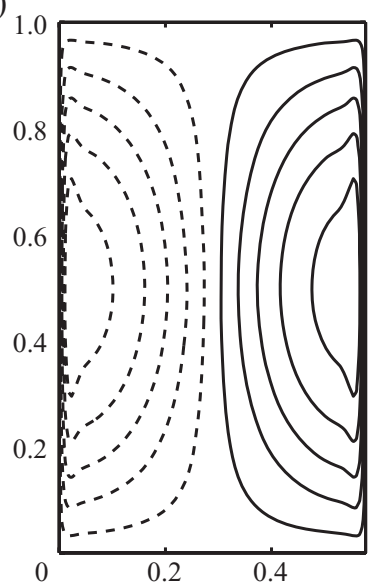

(i)

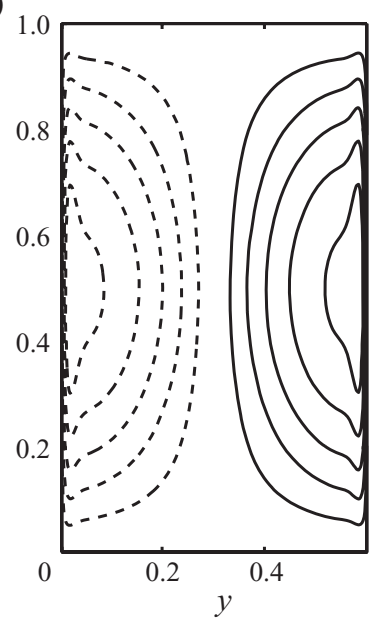

FIGURE 2. Contours of $(a) u_{x},(b) u_{y}$ and $(c) u_{z}$ in a vertical $y z$ plane computed from the asymptotic solution (3.38)-(3.40) (top); (d) $u_{x}$, (e) $u_{y}$ and $(f) u_{z}$ for the two-dimensional numerical solution (middle); $(g) u_{x},(h) u_{y}$ and $(i) u_{z}$ for direct three-dimensional nonlinear numerical simulation (bottom). The parameters for the three solutions are $\epsilon=5 \times 10^{-4}$, $\Gamma=1 / \sqrt{3}$ and $E=5 \times 10^{-5}$. 
three solutions obtained in completely different ways. Moreover, the kinetic energy, $E_{k i n}$, computed from the asymptotic expression (3.41) is $E_{k i n}=2.28 \times 10^{-5}$ for $\epsilon=5.0 \times 10^{-4}$ and $\Gamma=1 / \sqrt{3}$ at $E=5 \times 10^{-5}$ while the two-dimensional numerical analysis yields $E_{k i n}=2.13 \times 10^{-5}$ and the direct three-dimensional nonlinear simulation gives $E_{k i n}=2.01 \times 10^{-5}$. For a larger Poincaré number $\epsilon=10^{-3}$, expression (3.41) gives $E_{\text {kin }}=9.15 \times 10^{-5}$ for $\Gamma=1 / \sqrt{3}$ at $E=5 \times 10^{-5}$ while the corresponding twodimensional numerics yields $E_{k i n}=8.54 \times 10^{-5}$ and the direct three-dimensional nonlinear simulation produces a similar two-dimensional precessing flow with $E_{\text {kin }}=8.16 \times 10^{-5}$.

Our results again support the hypothesis that the viscous effect, which is primarily from the viscous boundary layers, is essential in determining both the structure and amplitude of weakly precessing flows at exact resonance. The asymptotic solution given by (3.38)-(3.40) offers a reasonably accurate approximation to the direct fully three-dimensional nonlinear simulation for the range $0<R e<O\left(E^{-1 / 2}\right)$. When $R e=O\left(E^{-1 / 2}\right)$, i.e. $\epsilon \approx 7 \times 10^{-3}$ for $E=5 \times 10^{-5}$, however, the nonlinear effect becomes important and leads to a fully three-dimensional precessing flow, which will be discussed later.

\subsection{Multiple-inertial-mode excitation at non-resonance}

Finally, consider a precessing channel with non-resonant aspect ratios, i.e. $\mid 3 n \Gamma-$ $\sqrt{3} j \mid \gg E^{1 / 2}$ for moderate integers $n$ and $j$, where $n$ is odd, at an asymptotically small $E$. In this case, it is anticipated that a large number of inertial modes would be excited by the precessional forcing. An asymptotic solution for a weakly precessing flow, $\boldsymbol{u}$ and $p$, can be still separated, at leading order, into the interior flow and the boundary-layer flow in the form

$$
\begin{aligned}
& \boldsymbol{u}(y, z, t)=\left[\left(\boldsymbol{u}_{G}+\widetilde{\boldsymbol{u}}_{G}\right)+\left(\sum_{j, n} \mathscr{C}_{j n} \boldsymbol{u}_{j n}\right)+\hat{\boldsymbol{u}}+\widetilde{\boldsymbol{u}}\right] \mathrm{e}^{\mathrm{i} t}, \\
& p(y, z, t)=\left[\left(p_{G}+\widetilde{p}_{G}\right)+\left(\sum_{j, n} \mathscr{C}_{j n} p_{j n}\right)+\hat{p}+\widetilde{p}\right] \mathrm{e}^{\mathrm{i} t},
\end{aligned}
$$

where $\mathscr{C}_{j n}$ are unknown complex coefficients, $\boldsymbol{u}_{G}$ and $\widetilde{\boldsymbol{u}}_{G}$ are the interior and boundary geostrophic flow, $\left(\boldsymbol{u}_{j n}, p_{j n}\right)$ represent inertial modes given by (3.7)-(3.10), $\widetilde{\boldsymbol{u}}$ is the viscous boundary layer while $[\hat{\boldsymbol{u}}, \hat{p}]$ represents the secondary interior flow with $|\hat{\boldsymbol{u}}| \ll\left|\sum_{j, n} \mathscr{C}_{j n} \boldsymbol{u}_{j n}\right|$. Note that $\boldsymbol{u}_{j n}$ and $p_{j n}$ in (3.42) and (3.43) are only a spatial function of $y$ and $z$ that is given by (3.7)-(3.10) without including the temporal dependence. We impose the non-slip boundary condition demanding that

$$
\hat{\boldsymbol{n}} \times\left[\widetilde{\boldsymbol{u}}+\sum_{j, n} \mathscr{C}_{j n} \boldsymbol{u}_{j n}\right]=0
$$

on the bounding surface of the channel while the normal component of the mass flux at the outer edge of the viscous boundary layer is

$$
\hat{\boldsymbol{n}} \cdot \hat{\boldsymbol{u}}=E^{1 / 2} \int_{0}^{\infty} \hat{\boldsymbol{n}} \cdot \nabla \times(\hat{\boldsymbol{n}} \times \widetilde{\boldsymbol{u}}) \mathrm{d} \xi .
$$




\begin{tabular}{|c|c|c|c|c|}
\hline$\Gamma$ & $\left|\overline{\mathscr{C}}_{j n}\right|$ & $\left|\overline{\mathscr{C}}_{j n}\right|$ & $\left|\overline{\mathscr{C}}_{j n}\right|$ & $\left|\overline{\mathscr{C}}_{j n}\right|$ \\
\hline $1 / \sqrt{3}$ & $\left|\overline{\mathscr{C}}_{11}\right|=7.45 \times 10^{-3}$ & $\left|\overline{\mathscr{C}}_{33}\right|=2.00 \times 10^{-4}$ & $\left|\overline{\mathscr{C}}_{12}\right|=1.33 \times 10^{-4}$ & $\left|\overline{\mathscr{C}}_{31}\right|=5.39 \times 10^{-5}$ \\
\hline 0.65 & $\left|\overline{\mathscr{C}}_{11}\right|=2.46 \times 10^{-3}$ & $\left|\overline{\mathscr{C}}_{31}\right|=1.19 \times 10^{-4}$ & $\left|\overline{\mathscr{C}}_{33}\right|=9.41 \times 10^{-5}$ & $\left|\overline{\mathscr{C}}_{51}\right|=5.28 \times 10^{-5}$ \\
\hline 1 & $\left|\overline{\mathscr{C}}_{11}\right|=7.40 \times 10^{-4}$ & $\left|\overline{\mathscr{C}}_{31}\right|=2.22 \times 10^{-4}$ & $\left|\overline{\mathscr{C}}_{53}\right|=1.38 \times 10^{-4}$ & $\left|\overline{\mathscr{C}}_{51}\right|=6.90 \times 10^{-5}$ \\
\hline$\sqrt{3}$ & $\left|\overline{\mathscr{C}}_{31}\right|=6.12 \times 10^{-3}$ & $\left|\overline{\mathscr{C}}_{11}\right|=5.84 \times 10^{-4}$ & $\left|\overline{\mathscr{C}}_{51}\right|=1.65 \times 10^{-4}$ & $\left|\overline{\mathscr{C}}_{32}\right|=1.08 \times 10^{-4}$ \\
\hline
\end{tabular}

TABLE 1. Four largest coefficients, $\overline{\mathscr{C}}_{j n}$, in the asymptotic expansion (3.42) and (3.43) computed numerically from the solvability condition (3.48) for several different values of $\Gamma$ with $E=5 \times 10^{-5}$ and $\epsilon=5 \times 10^{-4}$.

Substituting (3.42) and (3.43) into (3.1) and (3.2) and making use of (3.7)-(3.10), the secondary interior flow is described by

$$
\begin{aligned}
\mathrm{i} \hat{\boldsymbol{u}}+2 \hat{\boldsymbol{z}} \times \hat{\boldsymbol{u}}+\nabla \hat{p}= & \mathrm{i} \sum_{j, n}\left[\frac{2 n \Gamma-\left(j^{2}+n^{2} \Gamma^{2}\right)^{1 / 2}}{\left(j^{2}+n^{2} \Gamma^{2}\right)^{1 / 2}}\right] \mathscr{C}_{j n} \boldsymbol{u}_{j n} \\
& +E \sum_{j, n} \nabla^{2}\left(\mathscr{C}_{j n} \boldsymbol{u}_{j n}\right)+2 \epsilon z(\hat{\boldsymbol{x}}+\mathrm{i} \hat{\boldsymbol{y}}) \\
\nabla \cdot \hat{\boldsymbol{u}} & =0 .
\end{aligned}
$$

The solvability condition can be derived by multiplying (3.46) by $\boldsymbol{u}_{j n}^{*}$, the complex conjugate of $\boldsymbol{u}_{j n}$, making use of the asymptotic matching condition (3.45) and integrating the resulting equation over the channel, which yields a system of algebraic equations for $\mathscr{C}_{j n}$

$$
\begin{aligned}
& \mathscr{C}_{j n}\left\{\frac{\mathrm{i}\left[\left(j^{2}+n^{2} \Gamma^{2}\right)^{1 / 2}-2 n \Gamma\right]}{\left(j^{2}+n^{2} \Gamma^{2}\right)^{1 / 2}}+\frac{\pi^{2}\left(j^{2}+n^{2} \Gamma^{2}\right) E}{\Gamma^{2}}\right\} \int_{V}\left|\boldsymbol{u}_{j n}\right|^{2} \mathrm{~d} V \\
& +E^{1 / 2} \int_{S} p_{j n}^{*}\left[\int_{0}^{\infty} \hat{\boldsymbol{n}} \cdot \nabla \times(\hat{\boldsymbol{n}} \times \widetilde{\boldsymbol{u}}) \mathrm{d} \xi\right] \mathrm{d} S=2 \epsilon \int_{V} z \boldsymbol{u}_{j n}^{*} \cdot(\hat{\boldsymbol{x}}+\mathrm{i} \hat{\boldsymbol{y}}) \mathrm{d} V,
\end{aligned}
$$

where $n= \pm 1, \pm 3, \ldots, j=1,2 \ldots$ and $p_{j n}^{*}$ denotes the complex conjugate of $p_{j n}$. The general solvability condition (3.48) determines the value of coefficients $\mathscr{C}_{j n}$, which is valid for asymptotic solutions for $E \ll 1$ at any aspect ratio $\Gamma$. Note that because the mass flux from the viscous boundary layers couples all the inertial modes together, the algebraic equations resulting from (3.48) must be solved numerically. Plotted with many solutions obtained for different values of $\Gamma$ in the range $0<\Gamma \leqslant 2$, figure 3 shows kinetic energies of the precessing flows as a function of $\Gamma$ for rotating channels with four different values of $\epsilon$ at $E=5 \times 10^{-5}$. Table 1 gives values of the four largest coefficients $\left|\overline{\mathscr{C}}_{j n}\right|$ defined as

$$
\left|\overline{\mathscr{C}}_{j n}\right|=\left|\mathscr{C}_{j n}\right|\left[\int_{V}\left|\boldsymbol{u}_{j n}\right|^{2} \mathrm{~d} V\right]^{1 / 2},
$$

which is introduced because $\boldsymbol{u}_{j n}$ given by (3.8)-(3.10) is not normalized. At the principal resonance with $\Gamma=1 / \sqrt{3}$, the precessing flow is dominated by the inertial mode $\boldsymbol{u}_{11}$ with $\boldsymbol{u}_{33}$ making a small contribution, while the flow at the second resonance with $\Gamma=\sqrt{3}$ is dominated by the inertial mode $\boldsymbol{u}_{31}$. Even for a slight departure from the principal resonance - where the kinetic energy of the flow for $\epsilon=5 \times 10^{-4}$ drops from its resonant value $E_{k i n}=2.3 \times 10^{-5}$ at $\Gamma=1 / \sqrt{3}$ to $E_{k i n}=2.4 \times 10^{-6}$ at $\Gamma=0.65-$ the leading order of the precessing flow is still dominated by $\boldsymbol{u}_{11}$. For the non-resonant 


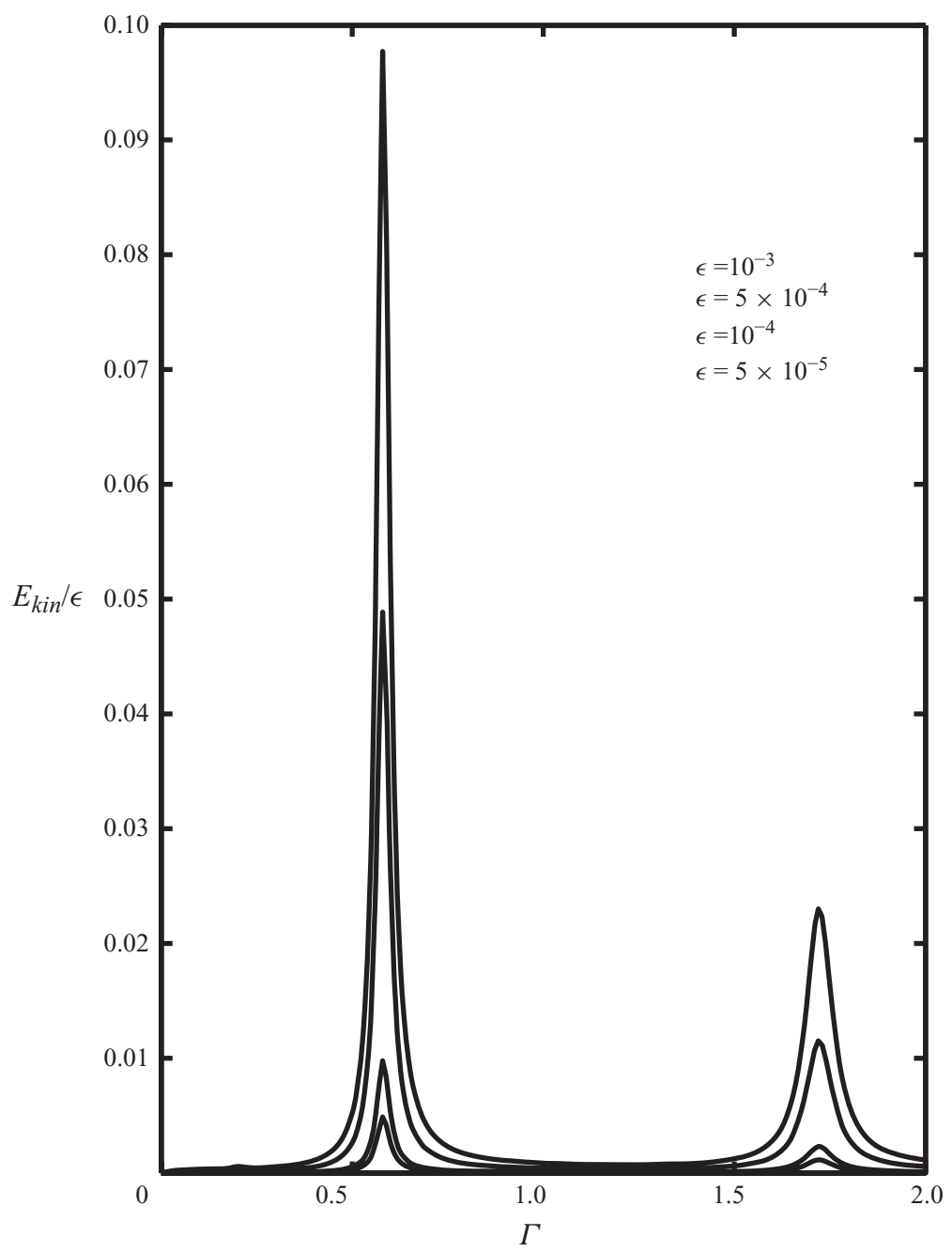

FIGURE 3. Kinetic energies of the precessing flow scaled by $\epsilon^{-1}$, resulting from solutions of the solvability condition (3.48), are plotted as a function of the aspect ratio $\Gamma$, in the range $0<\Gamma \leqslant 2$, in rotating channels at $E=5 \times 10^{-5}$ for four different values of $\epsilon$. The major resonances take place at $\Gamma=1 / \sqrt{3}$ and $\Gamma=\sqrt{3}$.

case $\Gamma=1$, however, the character of the precessing flow is quite different and several inertial modes that have comparable amplitudes are excited by the precessing forcing. It is significant to note that the number of inertial modes $\boldsymbol{u}_{j n}$ required in the asymptotic expansion (3.42) and (3.43) is determined by the aspect ratio $\Gamma$. This is why simple, explicitly analytical solutions like (3.24) are obtainable only for the resonant cases.

For any non-resonant aspect ratio with $|3 n \Gamma-\sqrt{3} j| \gg E^{1 / 2}$, however, the viscous effect in (3.48), represented by the terms proportional to $E$ and $E^{1 / 2}$, can safely be neglected. This approximation leads to a particularly simple system in which all inertial modes are completely decoupled. After carrying out the relevant integrations 
in (3.48), we find that

$$
\mathscr{C}_{j n}=\left[\frac{i 8 \Gamma \epsilon j^{2}}{n^{2} \pi^{4}\left(j^{2}+n^{2} \Gamma^{2}\right)}\right]\left\{\frac{\left[1-(-1)^{j}\right]\left[1-(-1)^{n}\right]}{\left[\left(j^{2}+n^{2} \Gamma^{2}\right)^{1 / 2}-n \Gamma\right]\left[\left(j^{2}+n^{2} \Gamma^{2}\right)^{1 / 2}-2 n \Gamma\right]}\right\} .
$$

Although the viscous boundary layer $\widetilde{\boldsymbol{u}}$, in contrast to the resonant cases, is not needed in the process of determining $\mathscr{C}_{j n}$, it is still required for describing a precessing flow that satisfies the non-slip boundary condition. An asymptotic solution with the nonslip boundary condition for a non-resonant channel can be written in the form

$$
\begin{aligned}
\boldsymbol{u}= & \widetilde{\boldsymbol{u}} \\
& +\left\{\frac{\left.4 \boldsymbol{u}_{G}+\widetilde{\boldsymbol{u}}_{G}\right)+\sum_{j, n}\left[\frac{\left[1-(-1)^{j}\right]\left[1-(-1)^{n}\right]}{n^{2} \pi^{3}\left(j^{2}+n^{2} \Gamma^{2}\right)^{1 / 2}}\right]}{\left[\left(j^{2}+n^{2} \Gamma^{2}\right)^{1 / 2}-n \Gamma\right]\left[\left(j^{2}+n^{2} \Gamma^{2}\right)^{1 / 2}-2 n \Gamma\right]}\right\} \\
& \times\left\{-\frac{\left(j^{2}+n^{2} \Gamma^{2}\right)^{1 / 2}}{j} \sin \frac{j \pi y}{\Gamma} \cos n \pi z\left[\hat{\boldsymbol{x}} \sin t+\hat{\boldsymbol{y}} \frac{n \Gamma \cos t}{\left(j^{2}+n^{2} \Gamma^{2}\right)^{1 / 2}}\right]\right. \\
& \left.+\hat{z}\left[\cos \frac{j \pi y}{\Gamma} \sin n \pi z \cos t\right]\right\},
\end{aligned}
$$

which is valid for an asymptotically small $E$ with non-resonant aspect ratios $\Gamma$. Note that the analytical expression (3.50) is non-singular because of the non-resonant condition. Of course, the summation must be truncated in any practical computation. It is found that the summation (3.50) with $j \leqslant O(10)$ and $|n| \leqslant O(10)$ would typically yield an accurate approximation within $1 \%$ error. In the asymptotic solution (3.50), while the geostrophic flow $\left(\boldsymbol{u}_{G}+\widetilde{\boldsymbol{u}}_{G}\right)$ is still given by (3.25), the viscous boundary layer $\widetilde{\boldsymbol{u}}$ is given by

$$
\begin{aligned}
\widetilde{\boldsymbol{u}}= & -\sum_{j, n} \frac{\mathrm{i} 2 \epsilon j^{2}\left[1-(-1)^{j}\right]\left[1-(-1)^{n}\right] \mathrm{e}^{\mathrm{i} t}}{n^{2} \pi^{3}\left(j^{2}+n^{2} \Gamma^{2}\right)\left[\left(j^{2}+n^{2} \Gamma^{2}\right)^{1 / 2}-n \Gamma\right]\left[\left(j^{2}+n^{2} \Gamma^{2}\right)^{1 / 2}-2 n \Gamma\right]} \\
& \times\left\{(-\hat{\boldsymbol{x}}+\mathrm{i} \hat{\boldsymbol{y}})\left[\frac{j\left(j^{2}+n^{2} \Gamma^{2}\right)^{1 / 2}}{\left(j^{2}+n^{2} \Gamma^{2}\right)^{1 / 2}+n \Gamma} \sin \frac{j \pi y}{\Gamma}\right] \exp \left(-\frac{\sqrt{6}(1+\mathrm{i}) z}{2 \sqrt{E}}\right)\right. \\
& -(\hat{\boldsymbol{x}}+\mathrm{i} \hat{\boldsymbol{y}})\left[\frac{j\left(j^{2}+n^{2} \Gamma^{2}\right)^{1 / 2}}{\left(j^{2}+n^{2} \Gamma^{2}\right)^{1 / 2}-n \Gamma} \sin \frac{j \pi y}{\Gamma}\right] \exp \left(-\frac{\sqrt{2}(1-\mathrm{i}) z}{2 \sqrt{E}}\right) \\
& +(-1)^{n}(-\hat{\boldsymbol{x}}+\mathrm{i} \hat{\boldsymbol{y}})\left[\frac{j\left(j^{2}+n^{2} \Gamma^{2}\right)^{1 / 2}}{\left(j^{2}+n^{2} \Gamma^{2}\right)^{1 / 2}+n \Gamma} \sin \frac{j \pi y}{\Gamma}\right] \exp \left(-\frac{\sqrt{6}(1+\mathrm{i})(1-z)}{2 \sqrt{E}}\right) \\
& -(-1)^{n}(\hat{\boldsymbol{x}}+\mathrm{i} \hat{\boldsymbol{y}})\left[\frac{j\left(j^{2}+n^{2} \Gamma^{2}\right)^{1 / 2}}{\left(j^{2}+n^{2} \Gamma^{2}\right)^{1 / 2}-n \Gamma} \sin \frac{j \pi y}{\Gamma}\right] \exp \left(-\frac{\sqrt{2}(1-\mathrm{i})(1-z)}{2 \sqrt{E}}\right) \\
& +\hat{z} \mathrm{i}\left[2\left(j^{2}+n^{2} \Gamma^{2}\right)^{1 / 2}\right] \sin n \pi z \\
& \left.\times\left[\exp \left(-\frac{\sqrt{2}(1+\mathrm{i}) y}{2 \sqrt{E}}\right)+(-1)^{j} \exp \left(-\frac{\sqrt{2}(1+\mathrm{i})(\Gamma-y)}{2 \sqrt{E}}\right)\right]\right\}
\end{aligned}
$$

It should be mentioned that we have carefully compared the numerical solution for (3.48), which contains all the viscous terms, with the explicit analytical solution (3.50) for $E \ll 1$ : the difference between them is negligibly small at a non-resonant $\Gamma$. On the basis of the explicit asymptotic solution (3.50), we can also derive an analytical 

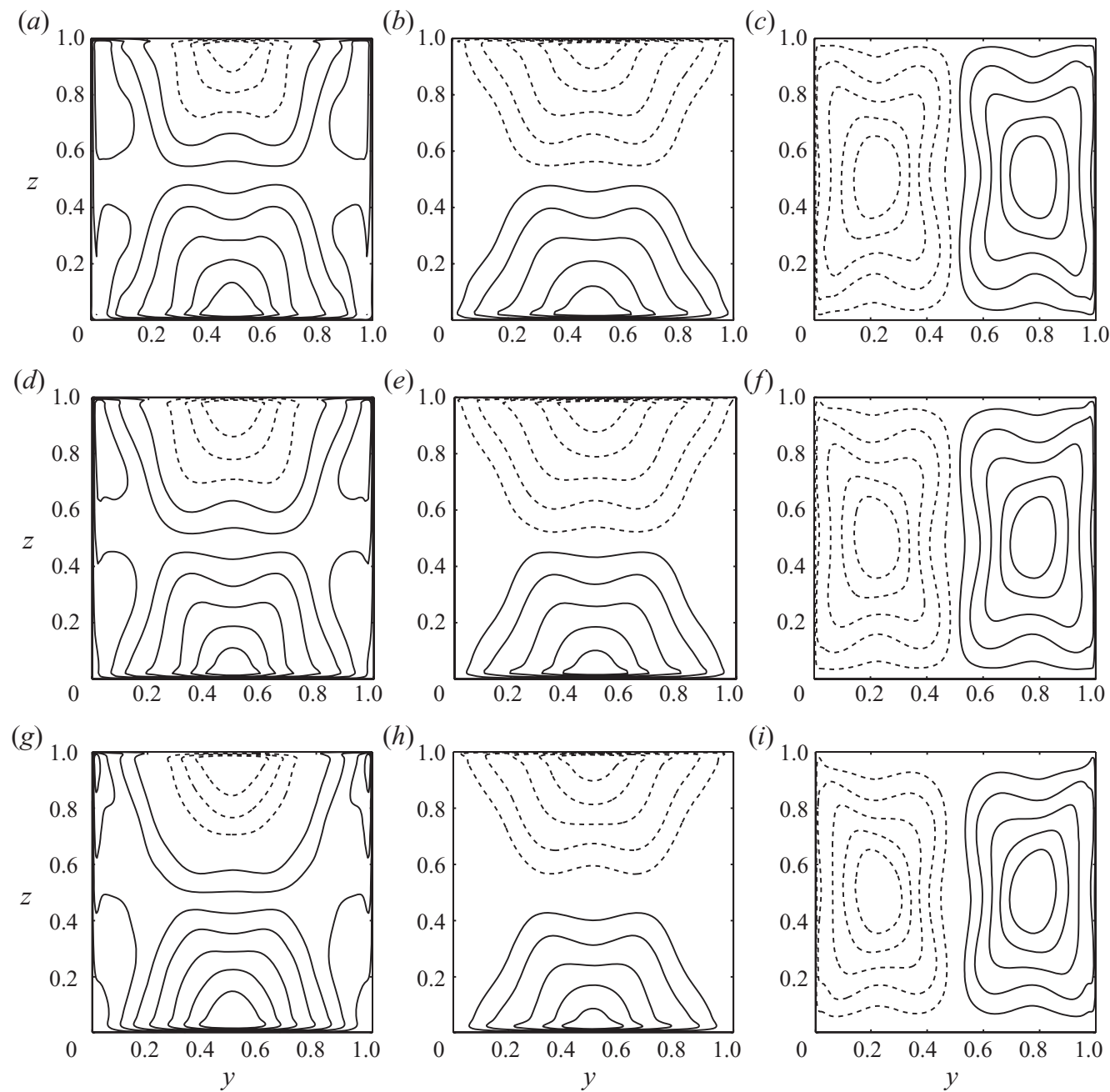

(h)
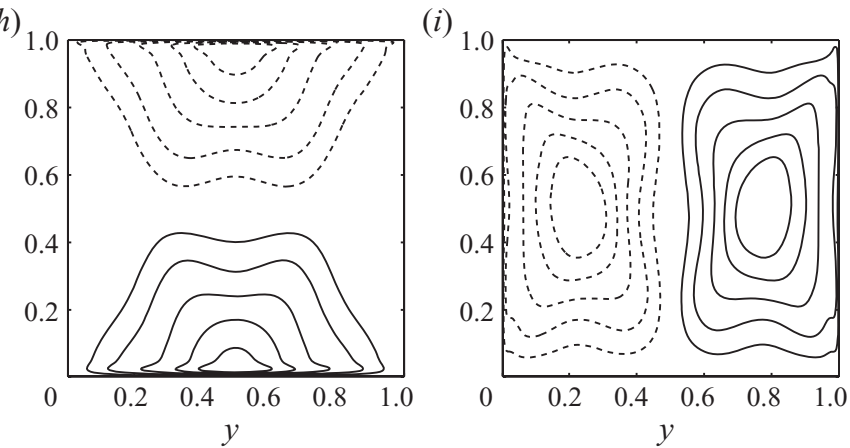

FIgURE 4. Contours of $(a) u_{x},(b) u_{y}$ and $(c) u_{z}$ in a vertical $y z$ plane from the non-resonant asymptotic solution (3.50) (top); $(d) u_{x},(e) u_{y}$ and $(f) u_{z}$ from the two-dimensional numerical analysis (middle); $(g) u_{x},(h) u_{y}$ and $(i) u_{z}$ from the three-dimensional direct simulation (bottom). The parameters are $\epsilon=5 \times 10^{-4}, \Gamma=1$ and $E=5 \times 10^{-5}$.

expression for the kinetic energy of the precessing flow

$$
E_{k i n}=\frac{\epsilon^{2}}{4}+\sum_{j, n} \frac{8 \epsilon^{2}\left[1-(-1)^{j}\right]\left[1-(-1)^{n}\right]}{n^{4} \pi^{6}\left[\left(j^{2}+n^{2} \Gamma^{2}\right)^{1 / 2}-n \Gamma\right]^{2}\left[\left(j^{2}+n^{2} \Gamma^{2}\right)^{1 / 2}-2 n \Gamma\right]^{2}},
$$

where the contribution from the boundary layers is again neglected.

For a given $\epsilon$ and non-resonant $\Gamma$, we can compute the spatial structure of a precessing flow satisfying the non-slip boundary condition from expression (3.50), which is shown in figure $4(a-c)$ for $\epsilon=5 \times 10^{-4}$ and $\Gamma=1$ at $E=5 \times 10^{-5}$. It can be then directly compared with the two-dimensional numerical solution shown in figure $4(d-f)$ and the three-dimensional nonlinear simulation in figure $4(g-i)$ at exactly the same parameters. There are no significantly noticeable differences in the spatial structure of the three solutions. Furthermore, kinetic energies, $E_{k i n}$, computed 


$\begin{array}{lcccc}\epsilon & R e & E_{\text {kin }} \text { (Asymptotic) } & E_{\text {kin }} \text { (2D numerics) } & E_{\text {kin }} \text { (3D simulation) } \\ 10^{-4} & 2 \times 10^{0} & 8.666 \times 10^{-9} & 8.670 \times 10^{-9} & 8.616 \times 10^{-9} \text { (2D flow) } \\ 5 \times 10^{-4} & 1 \times 10^{1} & 2.167 \times 10^{-7} & 2.168 \times 10^{-7} & 2.152 \times 10^{-9} \text { (2D flow) } \\ 10^{-3} & 2 \times 10^{1} & 8.666 \times 10^{-7} & 8.670 \times 10^{-7} & 8.603 \times 10^{-7} \text { (2D flow) } \\ 5 \times 10^{-3} & 1 \times 10^{2} & 2.167 \times 10^{-5} & 2.168 \times 10^{-5} & 2.151 \times 10^{-5} \text { (2D flow) } \\ 10^{-2} & 2 \times 10^{2} & 8.666 \times 10^{-5} & 8.670 \times 10^{-5} & 8.563 \times 10^{-5} \text { (2D flow) } \\ 5 \times 10^{-2} & 1 \times 10^{3} & 2.167 \times 10^{-3} & 2.168 \times 10^{-3} & 2.189 \times 10^{-3} \text { (2D flow) } \\ 7.5 \times 10^{-2} & 1.5 \times 10^{3} & & & \text { (3D flow) }\end{array}$

TABLE 2. Kinetic energies, $E_{k i n}$, computed from the asymptotic expression (3.52), along with the corresponding values calculated from the two-dimensional (2D) numerical analysis and direct three-dimensional (3D) nonlinear numerical simulation for $\Gamma=1$ and $E=5 \times 10^{-5}$.

from the analytical formula (3.52) for several different values of $\epsilon$ at $\Gamma=1$ are given in table 2, along with the corresponding values calculated from the two-dimensional and three-dimensional numerical analyses. Evidently, the asymptotic solution (3.50) offers an accurate approximation to the fully three-dimensional nonlinear simulation up to $R e \leqslant O\left(10^{3}\right)$, which corresponds to $\epsilon=5 \times 10^{-2}$ for $E=5 \times 10^{-5}$. When $R e$ increases further, the precessing flow becomes fully three-dimensional and the asymptotic solution (3.50) becomes physically irrelevant.

\section{Numerical simulation}

\subsection{Two-dimensional linear numerical analysis}

We first undertake a linear numerical analysis that is valid for any value of the Ekman number $E$ and any size of the aspect ratio $\Gamma$. The primary purpose of the analysis is to check the accuracy of our asymptotic analysis valid only for $E \ll 1$. Because the boundary condition is assumed to be no-slip, the numerical solution in a channel is quite complicated: the method of separation of variables fails and, consequently, a set of partial differential equations must be solved numerically. In the numerical analysis, we may expand a two-dimensional velocity in terms of two potentials, $\Psi$ and $\Phi$, in the form

$$
\boldsymbol{u}=\{\nabla \times[\Psi(y, z) \hat{\boldsymbol{x}}]+\Phi(y, z) \hat{\boldsymbol{x}}\} \mathrm{e}^{\mathrm{i} t},
$$

the real part of which will be taken as a solution of the problem. Substituting expression (4.1) into (3.1) and then applying $\hat{\boldsymbol{x}}$ and $\hat{\boldsymbol{x}} \cdot \nabla \times$, we can derive two independent partial differential equations governing a weakly precessing flow:

$$
\begin{aligned}
& 2 \epsilon z=-2 \frac{\partial \Psi}{\partial z}+\left[\mathrm{i}-E\left(\frac{\partial^{2}}{\partial y^{2}}+\frac{\partial^{2}}{\partial z^{2}}\right)\right] \Phi, \\
& 2 \mathrm{i} \epsilon=+2 \frac{\partial \Phi}{\partial z}+\left[\mathrm{i}-E\left(\frac{\partial^{2}}{\partial y^{2}}+\frac{\partial^{2}}{\partial z^{2}}\right)\right]\left(\frac{\partial^{2}}{\partial y^{2}}+\frac{\partial^{2}}{\partial z^{2}}\right) \Psi .
\end{aligned}
$$

The no-slip boundary condition in terms of $\Psi$ and $\Phi$ becomes

$$
\begin{aligned}
& \Psi=\Phi=\frac{\partial \Psi}{\partial z}=0 \quad \text { at } z=0,1, \\
& \Psi=\Phi=\frac{\partial \Psi}{\partial y}=0 \quad \text { at } y=0, \Gamma .
\end{aligned}
$$


Equations (4.2) and (4.3) are solved by making use of the Galerkin-type expansion:

$$
\begin{aligned}
& \Phi(y, z)=\sum_{l=0}^{N} \sum_{k=0}^{N} \widehat{\Phi}_{k l}\left[\left(1-\hat{z}^{2}\right) T_{l}(\hat{z})\right]\left[\left(1-\hat{y}^{2}\right) T_{k}(\hat{y})\right], \\
& \Psi(y, z)=\sum_{l=0}^{N} \sum_{k=0}^{N} \widehat{\Psi}_{k l}\left[\left(1-\hat{z}^{2}\right)^{2} T_{l}(\hat{z})\right]\left[\left(1-\hat{y}^{2}\right)^{2} T_{k}(\hat{y})\right],
\end{aligned}
$$

where $N$ is a truncation parameter taken to be of $O(100), \hat{y}=(2 y / \Gamma-1), \hat{z}=(2 z-1)$, $\hat{\Psi}_{k l}$ and $\hat{\Phi}_{k l}$ are complex coefficients and $T_{l}(x)$ denotes the standard Chebyshev functions. Substituting (4.6) and (4.7) into (4.2) and (4.3), we obtain a system of algebraic equations for the unknown coefficients. The resulting equations are then solved numerically by an iterative scheme to determine the coefficients, and hence the velocity potential $\Phi$ and $\Psi$ of a precessing flow.

An extensive numerical computation is carried out over a wide range of $E, \epsilon$ and $\Gamma$, some of which are shown in figures 1,2 and 4 as well as in table 2 . We can draw two conclusions from the two-dimensional numerical analysis: (i) the three different asymptotic expressions - (3.24) for $\Gamma=\sqrt{3}$, (3.38) for $\Gamma=1 / \sqrt{3}$ and (3.50) for non-resonant $\Gamma$-provide an accurate approximation to the numerical solutions for sufficiently small Ekman number with $E \leqslant O\left(10^{-3}\right)$ and (ii) consistent with the result of the asymptotic analysis, the amplitude of weakly precessing flows for a given $\epsilon$ and $E$ reaches an overall maximum at the double-mode resonance when $\Gamma=1 / \sqrt{3}$. How and when a precessionally driven two-dimensional flow becomes fully threedimensional at a large Reynolds number remains an open question that must be answered by a direct three-dimensional nonlinear simulation of the problem.

\subsection{Fully three-dimensional nonlinear simulation}

In comparison to the plane-geometry problem (Mason \& Kerswell 2002), which permits periodic boundary conditions in both the $x$ - and $y$-directions, the precessional problem in channel geometry with the non-slip boundary condition is numerically more challenging. It would be difficult, because of the existence of the two vertical sidewalls, to employ the widely used pseudo-spectral method with Fourier series. We choose to tackle the fully three-dimensional, nonlinear problem by employing a second-order finite-difference method based on the Chorin-type projection scheme (Chorin 1968), which decouples the momentum and continuity equations. The projection scheme leads to the time discretization of (2.4) and (2.5) in the form

$$
\frac{\left(\boldsymbol{u}_{m}-\boldsymbol{u}^{n}\right)}{\Delta t}=E \nabla^{2} \boldsymbol{u}^{n}-\boldsymbol{u}^{n} \cdot \nabla \boldsymbol{u}^{n}-2 \hat{z} \times \boldsymbol{u}^{n}+2 \epsilon\left(z+\boldsymbol{u}^{n} \times\right)\left(\hat{\boldsymbol{x}} \cos t_{n}-\hat{\boldsymbol{y}} \sin t_{n}\right),
$$

where $\boldsymbol{u}^{n}$ represents the velocity at the $n$th time step $t=t_{n}$ while $\boldsymbol{u}_{m}$ denotes the velocity at an intermediate time between $t=t_{n}$ and $t=t_{n+1}$. The solution of (4.8) gives rise to the intermediate velocity $\boldsymbol{u}_{m}$, which is then used to solve the Poisson equation for the pressure $p^{n+1}$ at the $(n+1)$ th time step $t=t_{n+1}$,

$$
\nabla^{2} p^{n+1}=\frac{1}{\Delta t} \nabla \cdot \boldsymbol{u}_{m}
$$

After solving (4.9), we can readily compute the velocity field $\boldsymbol{u}^{n+1}$ at $t=t_{n+1}$ by

$$
\boldsymbol{u}^{n+1}=\boldsymbol{u}_{m}-\Delta t \nabla p^{n+1} .
$$

No spatial symmetries are imposed on our direct three-dimensional numerical simulation, which usually starts with an arbitrary three-dimensional initial condition, 
even though the nonlinear solution for moderate values of $R e$ is not only twodimensional (independent of $x$ ) but also possesses spatial symmetries in the $y$ - and $z$-directions. Our numerical method is largely similar to that used by $\mathrm{Wu} \&$ Roberts (2008) although their time integration is a third-order scheme while our scheme is of second order.

In addition to the physical parameters like the Ekman number $E$ and the Poincaré number $\epsilon$, a new parameter enters into the three-dimensional nonlinear simulation: the size of the computational box defined by the upper and lower non-slip surfaces at $z=0,1$, by the two vertical non-slip sidewalls at $y=0, \Gamma$ and by the periodic boundary condition at $x=0, L$, where $L$ may be regarded as an additional parameter for the three-dimensional simulation. A thin computational box with $L \ll 1$ is computationally inexpensive, but it may be incapable of capturing the crucial spatial scales of three-dimensional instabilities; a very wide box with $L \gg 1$, on the other hand, is computationally too expensive or may be unnecessary in the understanding of key nonlinear dynamics. As a balance between the computational cost and the essential physics, we choose $L=1$, which equivalently imposes the periodic boundary conditions $\boldsymbol{u}(x=0)=\boldsymbol{u}(x=1)$ and $p(x=0)=p(x=1)$, for all our direct three-dimensional nonlinear simulation.

While our three-dimensional numerical code was carefully validated by comparing with both the constructed exact solution and the asymptotic solutions, the accuracy of the three-dimensional simulation was also carefully checked by computing the nonlinear solutions at exactly the same parameters but using different spatial and temporal resolutions. Note that we do not anticipate, physically and mathematically, boundary-layer-type structure in the $x$-direction along the channel, implying that $|\partial / \partial x| \ll|\partial / \partial z|$ and $|\partial / \partial x| \ll|\partial / \partial y|$. Our primary concern is with the viscous boundary layers located on the four bounding surfaces of a channel, in connection with the spatial resolution in the $y$ - and $z$-directions. It is found that there are no significant differences between the nonlinear solutions simulated with either 300 or 200 grids in the $z$-direction for $E \geqslant 5 \times 10^{-5}$. Consequently, we have typically used, for example at $\Gamma=\sqrt{3}$, the spatial resolution with a grid system $[50 \times 350 \times 200]$ covering $0 \leqslant x \leqslant 1,0 \leqslant y \leqslant \Gamma$ and $0 \leqslant z \leqslant 1$ for our direct three-dimensional simulations, providing a reasonable numerical accuracy for the moderately strong nonlinear solutions presented in this paper.

The finite-difference equations (4.8)-(4.10), treated as an initial-value problem, are integrated forward in time, starting from an arbitrary three-dimensional flow, until the numerical solution attains an oscillatory or a chaotic state, usually taking up to $O\left(10^{2}\right)$ non-dimensional time units. While a three-dimensional simulation for a two-dimensional weakly nonlinear flow is, because of relatively large $\Delta t$, usually less expensive, simulating a strongly nonlinear flow, however, would typically take a three-week runtime to integrate (4.8)-(4.10), with $\Delta t=O\left(10^{-4}\right)$, to the $O\left(10^{2}\right)$ time units using 60 processors (each processor with $3612 \mathrm{MHz}$ ) on an IBM parallel computer.

Consider first the exact resonance for $\Gamma=1 / \sqrt{3}$ at which the strongest precessing flow occurs for a given $\epsilon$. The primary objectives of our direct three-dimensional nonlinear simulation are twofold: to check the validity of the asymptotic expression (3.38) for moderate Reynolds numbers and to identify the transition regimes from twodimensional laminar flow to three-dimensional turbulence for large Reynolds numbers at fixed small Ekman number $E=5 \times 10^{-5}$. For a small or moderate value of $R e$, although a numerical simulation starts with an arbitrary three-dimensional initial flow, the final solution after the transient period of the simulation always reaches an 

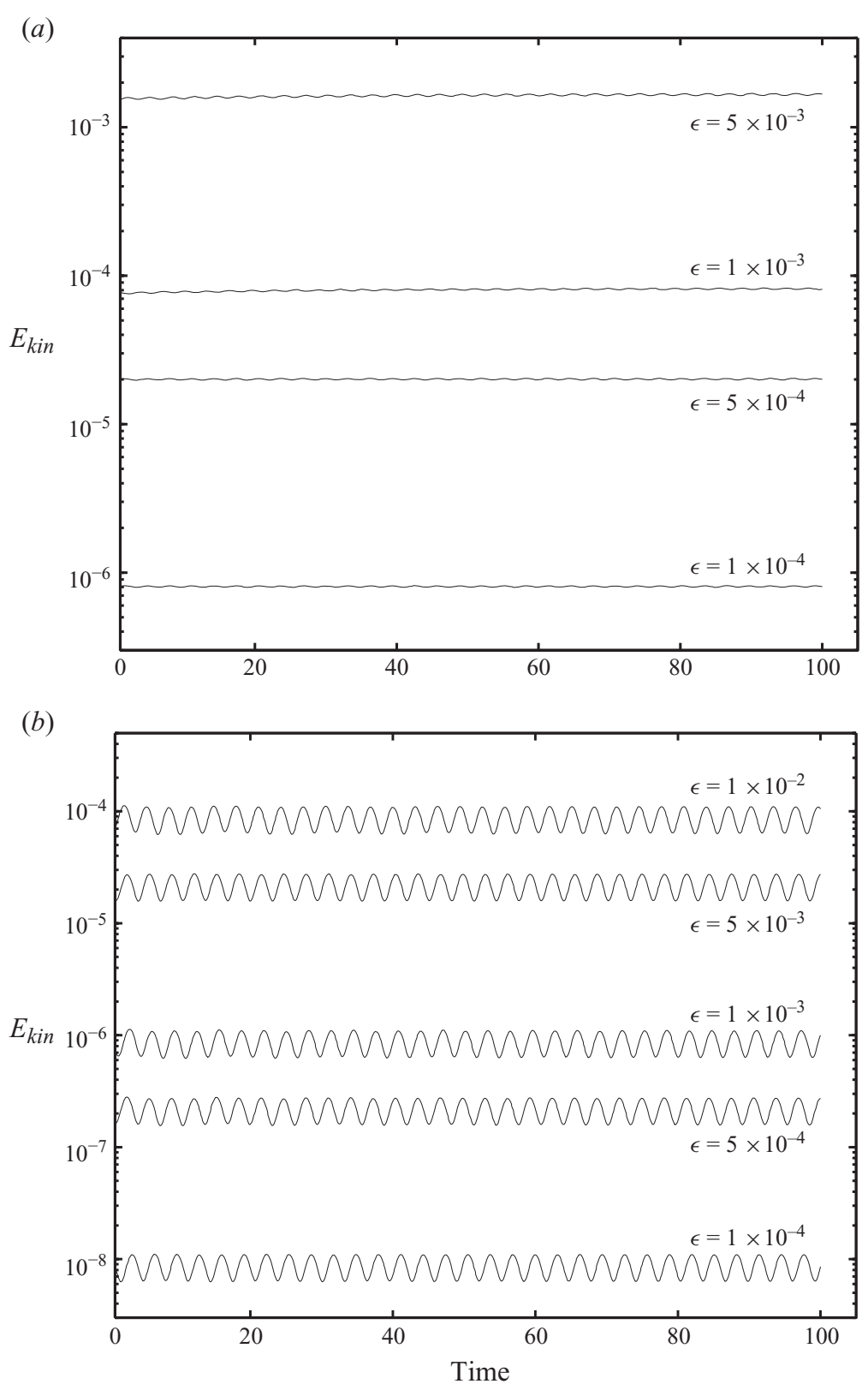

FIGURE 5. Kinetic energies, $E_{k i n}$, of the two-dimensional precessing flow resulting from three-dimensional direct numerical simulation are plotted against time for several different values of $\epsilon:(a)$ for the strongly resonant case with $\Gamma=1 / \sqrt{3}$ at $E=5 \times 10^{-5}$ and $(b)$ for the non-resonant case with $\Gamma=1$ at $E=5 \times 10^{-5}$.

$x$-independent state, i.e. $\partial \boldsymbol{u} / \partial x=0$ and $\partial p / \partial x=0$. Different simulations with various values of $L$, either $L>1$ or $L<1$, confirm that the $x$-independent nonlinear solution is the only physically realizable flow for small and moderate Reynolds numbers. The time dependence of the two-dimensional precessing flows resulting from the threedimensional nonlinear simulation is displayed in figure $5(a)$ with $\Gamma=1 / \sqrt{3}$. Because the initial transient period of a nonlinear simulation depends upon an arbitrary initial 
condition and, hence, is of less significance, only the final state of the simulation is shown in the figure. A typical structure of the weakly precessing flow obtained from the three-dimensional simulation is depicted in figure $2(g-i)$, along with the asymptotic solution given by (3.38). The result of the three-dimensional simulation shows a satisfactory agreement with the analytical expressions (3.38) and (3.41) in the approximate range $\left(\operatorname{Re} E^{1 / 2}\right)<O(1)$. This is consistent with the scaling provided by the asymptotic expression (3.38). The amplitude of the resonant precessing flow with $\Gamma=1 / \sqrt{3}$ is $|\boldsymbol{u}| \sim \epsilon / E^{1 / 2}$, giving rise to the nonlinear term $|\boldsymbol{u} \cdot \nabla \boldsymbol{u}| \sim \epsilon^{2} / E$ and the Coriolis force $|\hat{z} \times \boldsymbol{u}| \sim \epsilon / E^{1 / 2}$. It follows that, when the rotational effect is dominant, $|\hat{z} \times \boldsymbol{u}|>|\boldsymbol{u} \cdot \nabla \boldsymbol{u}|$ or $0<\left(R e E^{1 / 2}\right)<O(1)$, we would anticipate that the asymptotic solution (3.24) provides a reasonably good approximation and that the precessing flow remains two-dimensional. It reinforces the view that the viscous effect, which is primarily linked with the viscous boundary layers, determines the key property of weakly precessing flows at exact resonance. A large effort is then made to identify the critical value of $R e$ at which the two-dimensional precessing flow becomes fully three-dimensional in a channel with $\Gamma=1 / \sqrt{3}$. The scaling analysis suggests that the transition should occur when the nonlinear effect becomes significant at $R e E^{1 / 2}=O(1)$. By carrying out many three-dimensional simulations at different values of $R e$, we found that the instabilities that introduce the $x$-dependence and lead to a fully three-dimensional precessing flow take place at $\epsilon \approx 7.0 \times 10^{-3}$, or $\operatorname{Re} E^{1 / 2} \approx 1$ for $\Gamma=1 / \sqrt{3}$ and $E=5 \times 10^{-5}$. Figure 6(a) shows the time dependence of the threedimensional precessing flows for three different $R e$, where the transient behaviour is not displayed. In the range $1.5 \times 10^{2} \leqslant R e \leqslant 5 \times 10^{2}$, the nonlinear precessing flow is fully three-dimensional but remains largely laminar. A long time-scale temporal modulation can be clearly seen in figure $6(a)$, while the spatial modulation in the $x$-direction is shown in figure 7 for $\epsilon=7.5 \times 10^{-3}$ or $R e=1.5 \times 10^{2}$. When $R e$ increases to about $R e=10^{3}$, however, the laminar three-dimensional flow breaks down, leading to the small-scale disordered turbulence shown in figure 8. In short, three different regimes are identified with increasing $R e$ : (i) two-dimensional oscillatory and laminar flow that is in quantitative agreement with the analytical expression (3.38), (ii) threedimensional spatio-temporally modulated three-dimensional precessing flow and (iii) small-scaled disordered turbulence.

Finally, we consider a typical non-resonant case with $\Gamma=1$ also at $E=5 \times 10^{-5}$. In comparison with the resonant case $\Gamma=1 / \sqrt{3}$, our simulations starting with an arbitrary three-dimensional flow show that the precessing flows remain twodimensional, i.e. $\partial \boldsymbol{u} / \partial x=0 ; \partial p / \partial x=0$, within a much larger range $0<R e \leqslant 10^{3}$. A satisfactory quantitative agreement between the three-dimensional simulation and the analytical expression (3.50) is achieved for $0<R e \leqslant 10^{3}$, which is shown in table 2, where the physically preferred flow is two-dimensional, oscillatory and laminar. While the time-dependence of the two-dimensional oscillatory flow resulting from the threedimensional simulation is shown in figure $5(b)$ for $\Gamma=1$ and $E=5 \times 10^{-5}$, the typical spatial structure of the flow obtained from the three-dimensional nonlinear simulation is displayed in figure $4(g-i)$, along with the asymptotic solution given by (3.49). An extensive three-dimensional simulation for different values of $\epsilon$, which is shown in figure $6(b)$, indicates that the two-dimensional precessing flow becomes unstable to three-dimensional instabilities at $R e \approx 1.5 \times 10^{3} \quad\left(\epsilon=7.5 \times 10^{-2}\right)$, in contrast to $R e \approx 1.5 \times 10^{2}$ in the resonant case $\Gamma=1 / \sqrt{3}$. The strongly precessing flow at $R e=1.5 \times 10^{3}$ becomes fully three-dimensional and is spatially and temporally modulated. Its temporal modulation is shown in figure $6(b)$ while the spatial modulation in the $x$-direction is depicted in figure 9. When $\epsilon$ increases further 

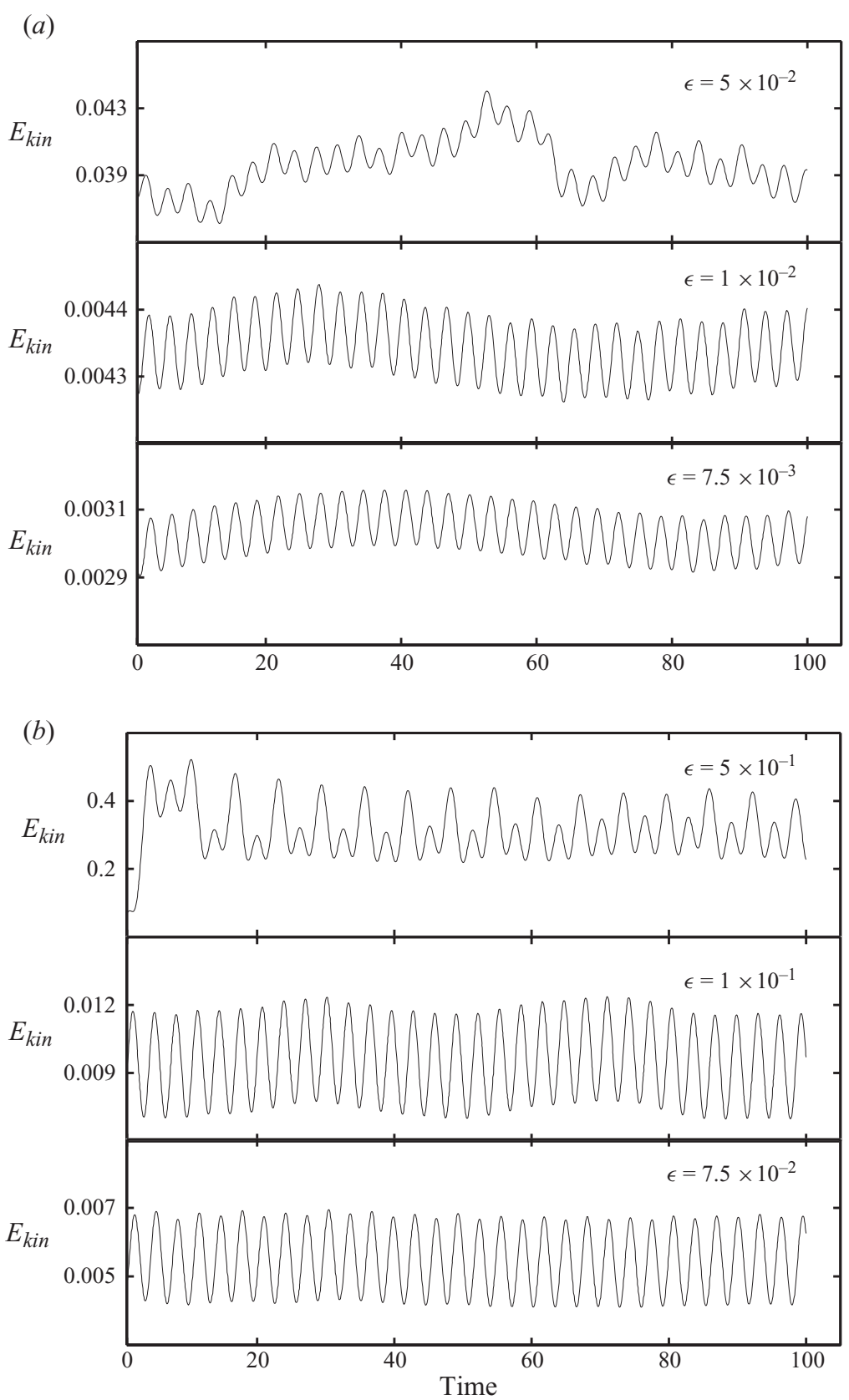

FIGURE 6. Kinetic energies, $E_{k i n}$, of the three-dimensional precessing flow from the three-dimensional direct numerical simulation are plotted against time $(a)$ for the resonant case with $\Gamma=1 / \sqrt{3}$ at $E=5 \times 10^{-5}$ and $(b)$ for the non-resonant case with $\Gamma=1$ at $E=5 \times 10^{-5}$.

to $5 \times 10^{-1}$, i.e. $R e=10^{4}$, the precessing flow breaks down, leading to the small-scale disordered flow which is shown in figure 10 .

There exist two major differences between the non-resonantly and resonantly precessing flows at exactly the same precessional rate. For resonantly precessing flows, the effect of viscosity, via both the Ekman boundary layers and interior viscous 
(a)

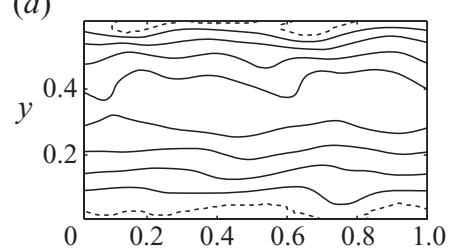

(b)

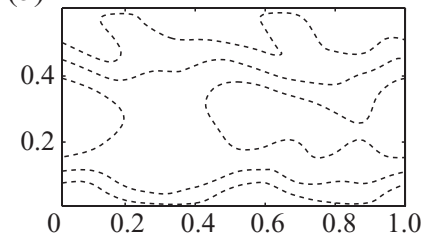

(d)

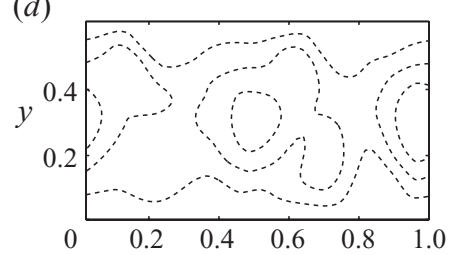

(e)

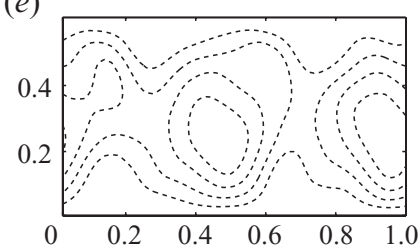

(c)

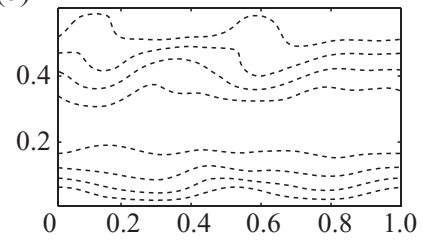

$(f)$

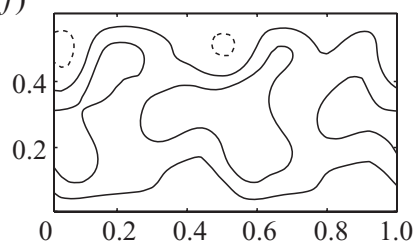

$(g)$

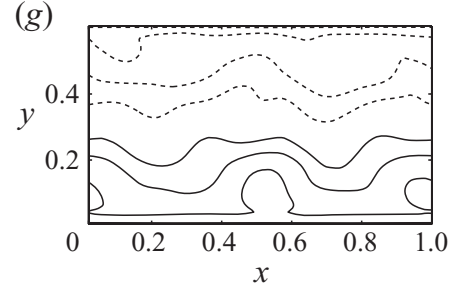

(h)

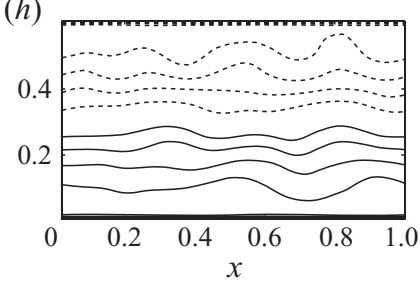

(i)

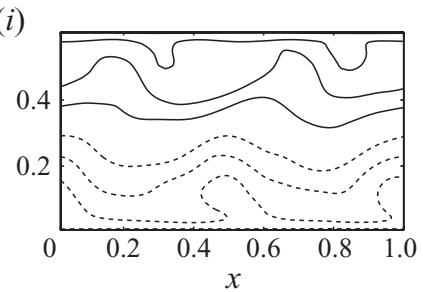

FIGURE 7. Snapshots of a three-dimensional precessing flow at three different instants in a horizontal $x y$ plane. Contours of $u_{x}$ at $z=1 / 4$ are displayed in $(a)-(c)$, contours of $u_{y}$ at $z=1 / 4$ are displayed in $(d)-(f)$, and contours of $u_{z}$ at $z=1 / 2$ are displayed in $(g)-(i)$. The parameters for this nonlinear solution are $\epsilon=7.5 \times 10^{-3}$ and $\Gamma=1 / \sqrt{3}$ at $E=5 \times 10^{-5}$.

(a)

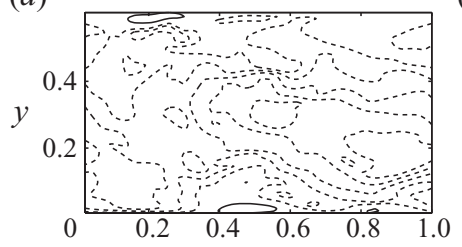

(d)

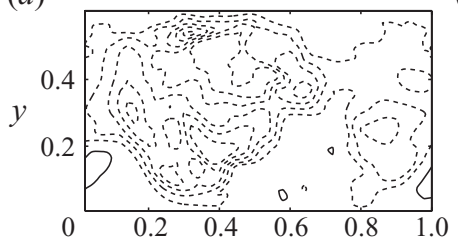

(g)

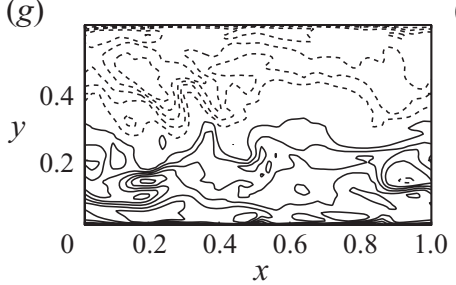

(b)

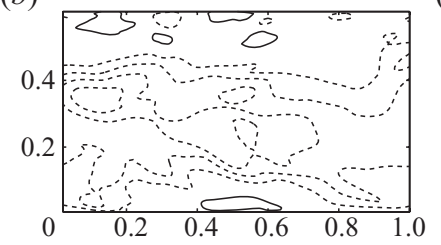

(e)

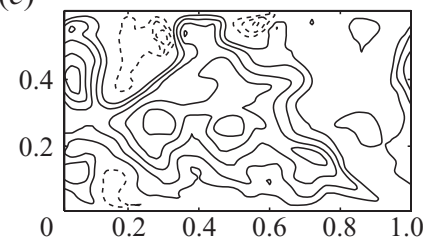

(h)

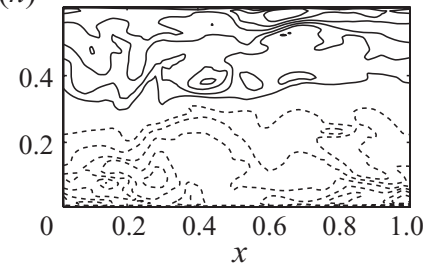

(c)

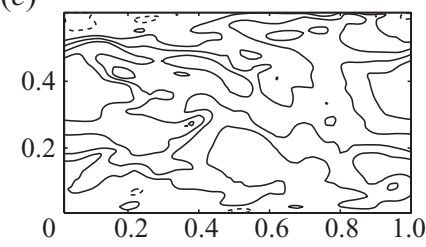

(f)

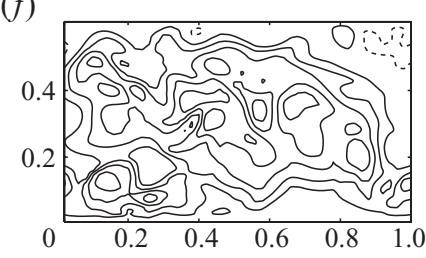

(i)

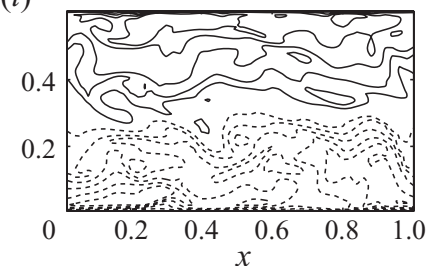

FIGURE 8. Snapshots of three-dimensional solutions in a horizontal $x y$ plane at three different instants. Contours of $u_{x}$ at $z=1 / 4$ are displayed in $(a)-(c)$, contours of $u_{y}$ at $z=1 / 4$ are displayed in $(d)-(f)$, and contours of $u_{z}$ at $z=1 / 2$ are displayed in $(g)-(i)$. The parameters for this nonlinear solution are $\epsilon=5 \times 10^{-2}$ and $\Gamma=1 / \sqrt{3}$ at $E=5 \times 10^{-5}$. 
(a)

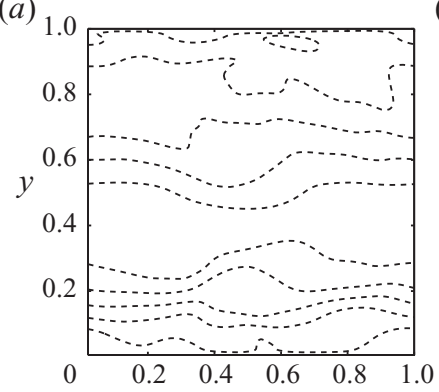

(d)

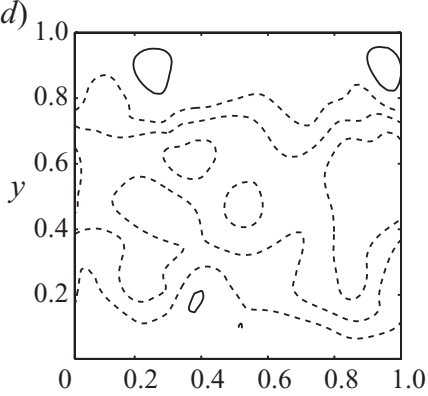

(g)

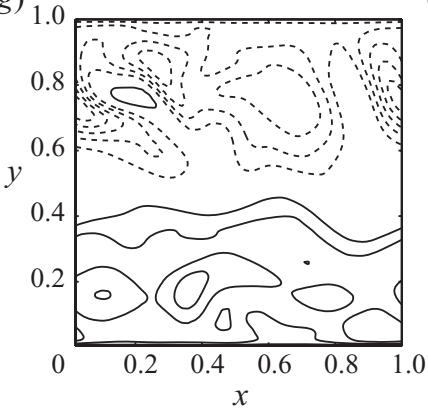

(b)

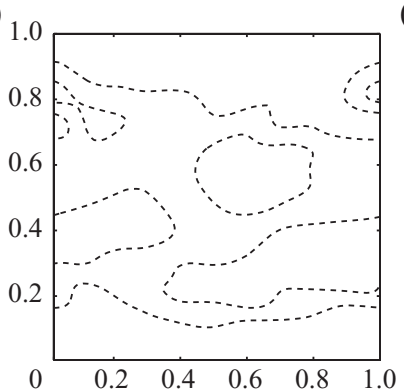

(c)

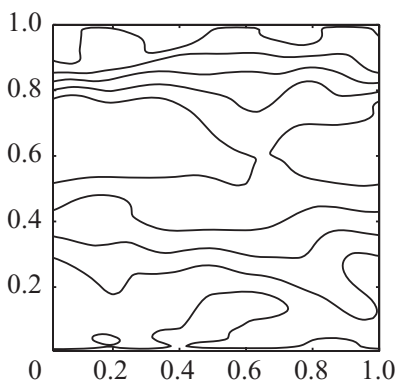

(e)

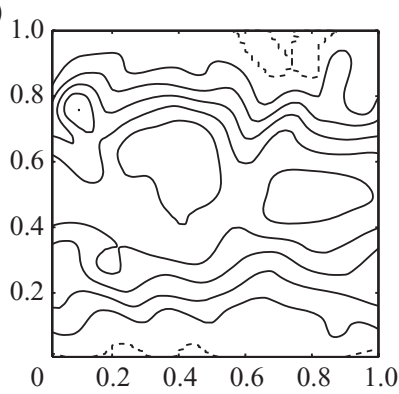

$(f)$

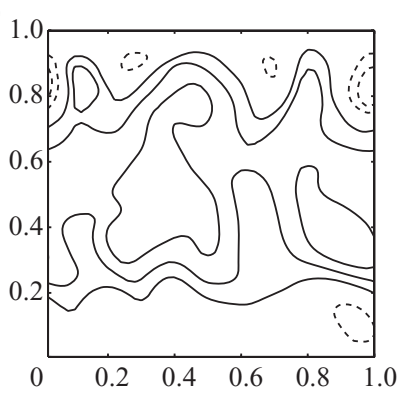

(h)

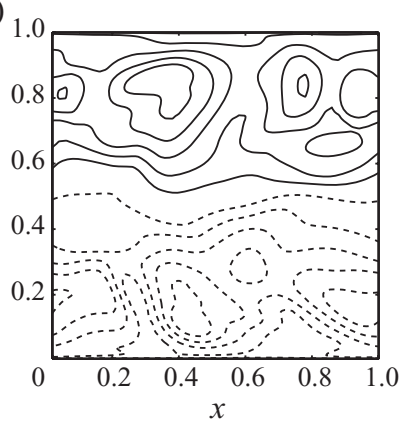

(i)

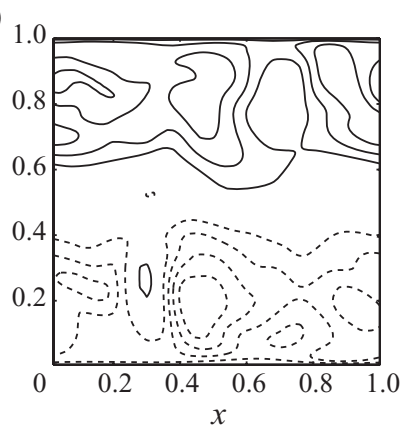

FIGURE 9. Snapshots of a three-dimensional precessing flow at three different instants in a horizontal $x y$ plane. Contours of $u_{x}$ at $z=1 / 4$ are displayed in $(a)-(c)$, contours of $u_{y}$ at $z=1 / 4$ are displayed in $(d)-(f)$, and contours of $u_{z}$ at $z=1 / 2$ are displayed in $(g)-(i)$. The parameters for this nonlinear solution are $\epsilon=7.5 \times 10^{-2}$ and $\Gamma=1$ at $E=5 \times 10^{-5}$.

effects, plays a critical role while, for non-resonantly precessing flows, the effect of viscosity is of secondary importance. The amplitude of the precessing flow at resonance is $O\left(\epsilon / E^{1 / 2}\right)$ with $E \ll 1$ while the amplitude of a non-resonant precessing flow is $O(\epsilon)$. It suggests the different underlying dynamics which is explicitly exhibited by the asymptotic expressions (3.38) and (3.50). Because the amplitude of the flow is dramatically enhanced by resonance, the critical value of $\epsilon$ at which the threedimensional turbulence sets in is hugely different between the non-resonantly and resonantly precessing flows.

\section{Summary and remarks}

We have studied, through both asymptotic analysis and numerical simulation, precessionally driven flows in a narrow annular channel that rotates rapidly about its axis of symmetry with angular velocity $\boldsymbol{\Omega}$ that itself precesses slowly about an axis 

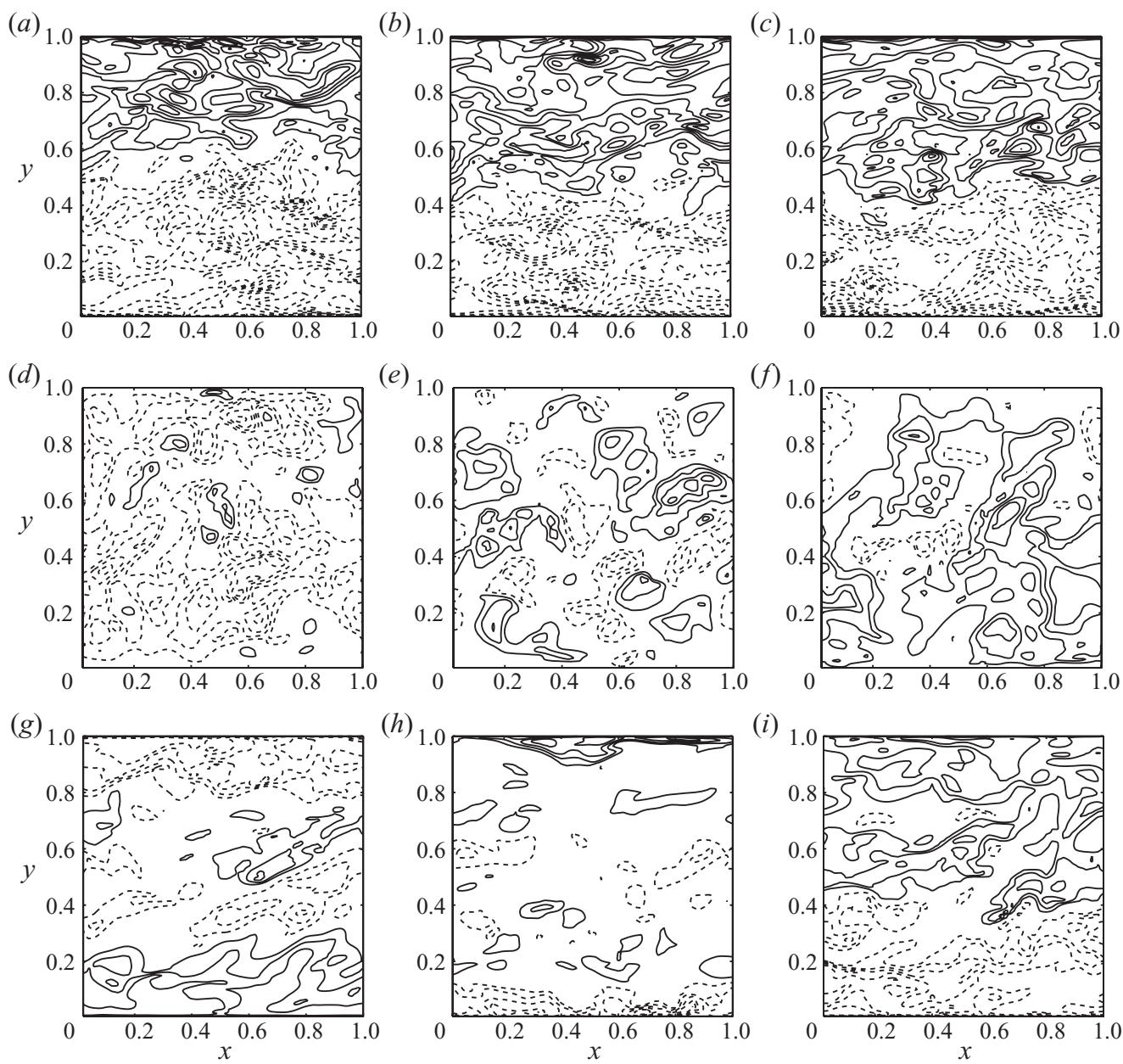

FIGURE 10. Snapshots of a three-dimensional precessing flow at three different instants. Contours of $u_{x}$ in a horizontal $x y$ plane at $z=1 / 4$ are displayed in $(a)-(c)$, contours of $u_{y}$ at $z=1 / 4$ are displayed in $(d)-(f)$, and contours of $u_{z}$ at $z=1 / 2$ are displayed in $(g)-(i)$. The parameters for this nonlinear solution are $\epsilon=5 \times 10^{-1}$ and $\Gamma=1$ at $E=5 \times 10^{-5}$.

fixed in an inertial frame. Not only is this precessional problem experimentally realizable (Davies-Jones \& Gilman 1971) but it also retains the mathematical simplicity and clarity of plane geometry. The essential asymptotic idea used for this study is in some ways similar to that developed for the convection problem in rotating fluid spheres (Zhang \& Liao 2004). Our asymptotic analysis reveals that, depending upon the aspect ratio $\Gamma$ of a channel, there exist three different regimes of precessing flows for which we have derived the three asymptotic expressions. At the single-inertial-mode resonance with $\Gamma=\sqrt{3}$ for which the contribution from higher inertial modes is insignificant and the viscous effect in connection with the viscous boundary layer largely controls the precessing flow, a simple analytical solution satisfying the non-slip boundary condition is given by the expression (3.24). At the double-inertial-mode resonance with $\Gamma=1 / \sqrt{3}$ for which the viscous effect also plays an essential role in controlling the amplitude of the precessing flow, a slightly more complicated expression satisfying the non-slip boundary condition is given by 
(3.38). For other values of $\Gamma$, non-resonant precessing flows are described by the analytical expression (3.50) satisfying all the required non-slip boundary conditions in which, however, the viscous effect no longer plays a critical role in determining the character and amplitude of the precessing flow. A satisfactory agreement between the three asymptotic solutions and the linear/nonlinear numerical simulation has been achieved for small and moderately large Reynolds numbers at an asymptotically small $E$.

Although it is inappropriate to make a direct comparison between the precessionally driven flows taking place in a precessing channel and in a precessing sphere (Roberts \& Stewartson 1965; Busse 1968), there exists a close analogy between the two precessing systems: the underlying dynamics/mechanism is, in many respects, similar. In the body (or mantle) frame of reference, the principal resonant mode in spherical geometry, $\left[p_{s p i n}, \boldsymbol{u}_{s p i n}\right]$, takes the form

$$
\begin{aligned}
p_{\text {spin }} & =\mathscr{C}_{\text {spin }} r^{2} \cos \theta \sin \theta \cos (\phi+t), \\
\hat{\boldsymbol{r}} \cdot \boldsymbol{u}_{\text {spin }} & =0, \\
\hat{\boldsymbol{\theta}} \cdot \boldsymbol{u}_{\text {spin }} & =\mathscr{C}_{\text {spin }} r \sin (\phi+t), \\
\hat{\boldsymbol{\phi}} \cdot \boldsymbol{u}_{\text {spin }} & =\mathscr{C}_{\text {spin }} r \cos \theta \cos (\phi+t),
\end{aligned}
$$

where $\mathscr{C}_{\text {spin }}$ denotes the amplitude of the mode, which is usually referred to as the spin-over mode (or the Poincare flow) (Greenspan 1968), where $\boldsymbol{u}_{\text {spin }}$ is its threedimensional velocity in spherical polar coordinates $(r, \theta, \phi)$ with $\theta=0$ at the axis of rapid rotation $\Omega \hat{z}$ and with the corresponding unit vectors $(\hat{\boldsymbol{r}}, \hat{\boldsymbol{\theta}}, \hat{\boldsymbol{\phi}})$. Note that, in the body frame of reference, the spin-over mode $\boldsymbol{u}_{\text {spin }}$ is just the lowest order of spherical inertial modes with the azimuthal wavenumber $m=1$ (Zhang et al. 2001) which satisfies

$$
\begin{gathered}
2 \mathrm{i} \sigma_{\text {spin }} \boldsymbol{u}_{\text {spin }}+2 \hat{z} \times \boldsymbol{u}_{\text {spin }}+\nabla p_{\text {spin }}=0, \\
\nabla \cdot \boldsymbol{u}_{\text {spin }}=0,
\end{gathered}
$$

where $\sigma_{\text {spin }}=1 / 2$ is the half-frequency of the spin-over mode, subject to the condition of vanishing normal flow

$$
\hat{\boldsymbol{n}} \cdot \boldsymbol{u}_{\text {spin }}=0
$$

at the bounding surface of the sphere. That is, $\boldsymbol{u}_{\text {spin }}$ satisfies exactly the same equations and the same inviscid boundary condition as the principal resonant mode $\boldsymbol{u}_{11}$ in channel geometry. In other words, the principal resonant mode $\boldsymbol{u}_{11}$ - the lowest order of channel inertial modes - represents the counterpart of spin-over mode $\boldsymbol{u}_{\text {spin }}$. In spherical geometry, it is the thin spherical Ekman boundary layer that determines the amplitude of $\boldsymbol{u}_{\text {spin }}$ with $\mathscr{C}_{\text {spin }}=O\left(\epsilon / E^{1 / 2}\right)$ (Roberts \& Stewartson 1965), while in channel geometry it is also the Ekman boundary layers on the four walls that determine the amplitude of the principal mode $\boldsymbol{u}_{11}$ with $\mathscr{C}_{11}=O\left(\epsilon / E^{1 / 2}\right)$. In spherical geometry, the spin-over mode, which interacts with other inertial modes nonlinearly, becomes unstable to three-dimensional instabilities at sufficiently large $\epsilon$ (Kerswell 1993; Tilgner \& Busse 2001). In channel geometry, the principal mode $\boldsymbol{u}_{11}$, which also interacts with higher-order inertial modes nonlinearly, becomes unstable to threedimensional instabilities at sufficiently large $\epsilon$. An important difference between the asymptotic solutions in channel and spherical geometries is perhaps the existence of the singular behaviour at critical latitudes in the spherical viscous boundary layer (Roberts \& Stewartson 1965) which is absent from the channel viscous boundary layers (where the singularities are lost in the corners of the channel where the boundary 
layers meet). This absence may be attributable to the result that the simple asymptotic solutions in a precessing channel provide a reasonably accurate approximation to the fully nonlinear three-dimensional simulation even for moderately large Reynolds numbers.

An interesting nonlinear phenomenon found in the strongly resonant precessing flow in a precessing channel is a sudden breakdown of the large-scale three-dimensional laminar flow to small-scale disordered turbulence. Our asymptotic and numerical analysis suggests the following transition scenario at exact resonance: the physically realizable flow in $0<R e<O\left(E^{-1 / 2}\right)$ is two-dimensional, oscillatory and laminar, which becomes unstable to three-dimensional instabilities at $R e=O\left(E^{-1 / 2}\right)$ leading a spatio-temporally modulated three-dimensional flow; when $R e$ increases slightly after the onset of the three-dimensional instability, the three-dimensional modulated flow suddenly collapses towards small-scaled disordered turbulence. Similar sudden breakdowns of the precessing flows were observed in various precessional experiments for spherical and cylindrical geometries (Gans 1970; Malkus 1989; Eloy, Le Gal \& Le Dizs 2003). The three-dimensional instabilities in a precessing channel may be caused by a similar mechanism to a triadic resonance discussed in a precessing cylinder where either the geostrophic mode in an infinite cylinder (Mahalov 1993) or the principal resonant mode in a finite cylinder (Kerswell 2002; Lagrange et al. 2008) resonates with two cylindrical inertial modes. However, a further theoretical study that includes explicitly the nonlinear interaction between the geostrophic mode and specific inertial modes is required to elucidate the precise mechanism of the instabilities in a precessing channel. With its rich dynamics demonstrated by our asymptotic analysis and numerical experiments, this opens an exciting new line of research of a laboratory experimental study on precessionally driven flows in a precessing annular channel.

K.Z. expresses his thanks to Professor P. H. Roberts for helpful discussions on the precessional problem and is supported by UK STFC/NERC grants and X.L. is supported by NSFC (10633030), STCSM (08XD14052) and CAS grants. The numerical computation is supported by WSC (Wuxi Supercomputer Centre).

\section{REFERENCES}

Bullard, E. C. 1949 The magnetic flux within the Earth. Proc. R. Soc. Lond. A 197, 433-453.

Busse, F. H. 1968 Steady fluid flow in a precessing spheroidal shell. J. Fluid Mech. 136, 739-751.

Busse, F. H. 1994 Convection driven zonal flows and vortices in the major planets. CHAOS 4, $123-134$.

Busse, F. H. 2005 Convection in a narrow annular channel rotating about its axis of symmetry. J. Fluid Mech. 537, 145-154.

Chorin, A. J. 1968 Numerical solutions of Navier-Stokes equations. Math. Comp. 22, 745-762.

Davies-Jones, R. P. \& Gilman P. A. 1971 Convection in a rotating annulus uniformly heated from below. J. Fluid Mech. 46, 65-81.

Eccles, F. J. R., Read, P. L., Castrejn-Pita, A. A. \& Haine, T. W. N. 2009 Synchronization of modulated travelling baroclinic waves in a periodically forced, rotating fluid annulus. Phys. Rev. E 79, 015202.

Eloy, C., Le Gal, P. \& Le Dizs, S. 2003 Elliptic and triangular instabilities in rotating cylinders. J. Fluid Mech. 476, 357-388.

Gans, R. F. 1970 On the precession of a resonant cylinder. J. Fluid Mech. 476, 865-872.

Gilman, P. A. 1973 Convection in a rotating annulus uniformly heated from below. Part 2. Nonlinear results. J. Fluid Mech. 57, 381-400.

Greenspan, H. P. 1968 The Theory of Rotating Fluids. Cambridge University Press. 
Hollerbach, R. \& Kerswell, R. R. 1995 Oscillatory internal shear layers in rotating and precessing flows. J. Fluid Mech. 298, 327-339.

Jones, C. A., Rotvig, J. \& Abdulrahman, A. 2003 Multiple jests and zonal flow on Jupiter. Geophys. Res. Lett. 30, 1-4.

Kerswell, R. R. 1993 The instability of precessing flow. Geophys. Astrophys. Fluid Dyn. 72, 107-144.

Kerswell, R. R. 1996 Upper bounds on the energy dissipation in turbulent precession. J. Fluid Mech. 321, 335-370.

Kerswell, R. R. 2002 Elliptical instability. Annu. Rev. Fluid Mech. 34, 83-113.

Kobine, J. J. 1995 Inerital wave dynamics in a rotating and precessing cylinder. J. Fluid Mech. 303, 233-252.

Lagrange, R., Eloy, C., Nadal, F. \& Meunier, P. 2008 Instability of a fluid inside a precessing cylinder. Phys. Fluids 20, 081701

Li, L., LiaO, X., Chan, K. H. \& Zhang, K. 2008 Linear and nonlinear instabilities in rotating cylindrical Rayleigh-Bénard convection. Phys. Rev. E 78, 056303-1-056303-12.

LiAO, X. \& ZHANG, K. 2009 Inertial oscillation, inertial wave and initial value problem in rotating annular channels. Geophys. Astrophys. Fluid Dyn. 103, 199-222.

LiaO, X., Zhang, K. \& Chang, Y. 2006 On boundary-layer convection in a rotating fluid layer. J. Fluid Mech. 549, 375-384.

Mahalov, A. 1993 The instability of rotating fluid columns subjected to a weak external Coriolis force. Phys. Fluids A 5, 891-900.

Malkus, W. V. R. 1968 Precession of the Earth as the cause of geomagnetism. Science 136, 259-264.

MaLKUS, W. V. R. 1989 An experimental study of global instabilities due to tidal (elliptical) distortion of a rotating elastic cylinder. Geophys. Astrophys. Fluid Dyn. 48, 123-134.

Manasseh, R. 1992 Breakdown regimes of inertia waves in a precessing cylinder. J. Fluid Mech. 243, 261-296.

Mason, R. M. \& Kerswell, R. R. 2002 Chaotic dynamics in a strained rotating flow: a precessing plane fluid layer. J. Fluid Mech. 471, 71-106.

Meunier, P., Eloy, C., Lagrange, R. \& Nadal, F. 2008 A rotating fluid cylinder subject to weak precession. J. Fluid Mech. 599, 405-440.

Noir, J., CARdin, P., JAULT, D. \& Masson J. P. 2003 Experimental evidence of nonlinear resonance effects between retrograde precession and the tilt-over mode within a spheroid. Geophys. J. Intl 154, 407-416.

Noir, J., JAULt, D. \& CARDin, P. 2001 Numerical study of the motions within a slowly precessing sphere at low Ekman number. J. Fluid Mech. 437, 283-299.

Roberts, P. H. \& Stewartson, K. 1965 On the motion of a liquid in a spheroidal cavity of a precessing rigid body. II. Math. Proc. Camb. Phil. Soc. 61, 279-288.

Tilgner, A. 1999 Magnetohydrodynamic flow in precessing spherical shells. J. Fluid Mech. 136, 303-318.

Tilgner, A. 2005 Precession driven dynamos. Phys. Fluids 17, 034104-034106.

Tilgner, A. 2007 Kinematic dynamos with precession driven flow in a sphere. Geophys. Astrophys. Fluid Dyn. 100, 1-9.

Tilgner, A. \& Busse, F. H. 2001 Fluid flows in precessing spherical shells. J. Fluid Mech. 426, 387-396

Vanyo, J. P. 1993 Rotating Fluids in Engineering and Science. General Publishing.

VAnyo, J. P., Wilde, P., CARdin, P. \& Olson, P. 1995 Experiments on precessing flows in the Earth's liquid core. Geophys. J. Intl 121, 136-142.

Wu, C. C. \& Roberts, P. H. 2008 A precesionally-driven dynamo in a plane layer. Geophys. Astrophys. Fluid Dyn. 102, 1-19.

Wu, C. C. \& Roberts, P. H. 2009 On a dynamo driven by topographic precession. Geophys. Astrophys. Fluid Dyn. 103, 467-501.

Zhang, K., Earnshaw, P., Liao, X. \& Busse, F. H. 2001 On inertial waves in a rotating fluid sphere. J. Fluid Mech. 437, 103-119.

ZhANG, K. \& LiAO, X. 2004 A new asymptotic method for the analysis of convection in a rotating sphere. J. Fluid Mech. 518, 319-346.

Zhang, K. \& Liao, X. 2008 On the initial value problem in a rotating circular cylinder. J. Fluid Mech. 610, 425-443. 